\title{
Diretrizes para a Cirurgia das Doenças da Aorta
}

\author{
Editores \\ Luciano Cabral Albuquerque, \\ José Honório Palma, \\ Domingo Braile \\ Revisor \\ Walter Gomes \\ Coordenador de Normatizações e Diretrizes \\ Jorge Ilha Guimarães
}




\section{Introdução}

A despeito dos avanços ocorridos nos exames diagnósticos, nos métodos de monitorização e suporte hemodinâmico, e nas técnicas de correção cirúrgica, as doenças da aorta continuam sendo importante causa de mortalidade e morbidade cardiovascular, e um permanente desafio a cardiologistas e cirurgiões.

Enquanto nos casos de dissecções agudas (DA), o consenso quanto à necessidade de cirurgia imediata esteja estabelecido, há pelo menos três décadas, no caso das doenças da aorta ascendente o adequado momento para intervenção nas doenças degenerativas, que determinam dilatação assintomática da aorta ascendente, bem como o manejo da raiz da aorta e da válvula aórtica, ainda constituem objeto de discussão e de inúmeras contribuições recentes na literatura.

No envolvimento do arco transverso, as controvérsias estão centradas na definição de quando e em que extensão o arco deve ser incluído na reconstrução e na escolha do melhor método de proteção cerebral, como a parada cardiocirculatória hipotérmica, a retroperfusão venosa, a perfusão cerebral seletiva e, mais recentemente, a perfusão anterógrada pelo eixo subclávio-axilar.

No tratamento das doenças da aorta descendente e tóraco-abdominal, a isquemia medular é ainda a principal preocupação, com ocorrência variável, porém significativa, nas diferentes abordagens descritas, sendo um dos motivadores do advento dos stents autoexpansíveis. Além disso, em casos de aneurismas degenerativos, à luz de estudos recentes sobre a predição da taxa de dissecção ou ruptura, a indicação de intervenção baseada, exclusivamente, no diâmetro tem dado lugar a decisões mais individualizadas, que consideram fatores como idade, condições co-mórbidas e resultados do grupo cirúrgico, entre outros.

$\mathrm{Na}$ aorta abdominal, a indicação clássica de correção cirúrgica dos aneurismas infra-renais assintomáticos, a partir de $5 \mathrm{~cm}$ de diâmetro, pode estar sendo modificada pelos resultados de recentes ensaios europeus e norte-americanos; igualmente, a experiência com a exclusão endoluminal pelo uso de stents, descrita em diversos estudos que comparam esta técnica à cirurgia aberta, tem sido alvo de discussão, e ainda está distante de um consenso.

Estas Diretrizes têm o propósito de revisar, criticamente, as indicações e os resultados cirúrgicos no tratamento das diversas doenças da aorta, utilizando a grade de evidências, proposta pela American Heart Association/ American College of Cardiology, assim classificada:

Classe I: situação em que existem evidências e/ou concordância geral de que o procedimento é benéfico e efetivo.

Classe II: situação em que existem evidências conflitantes e/ou divergentes sobre a utilidade e eficácia do procedimento ou tratamento.

Classe IIa: situação em que o peso das evidências e opiniões favorece a utilização do procedimento ou tratamento.

Classe IIb: situação em que a utilização e a eficácia do procedimento ou tratamento não estão bem suportadas pelas evidências e opiniões.
Classe III: situação em que existem evidências e/ou concordância geral de que o procedimento ou o tratamento não é benéfico, podendo até ser deletério.

Nível de evidência $\mathrm{A}$ : dados obtidos a partir de vários ensaios randomizados ou de meta-análises de ensaios clínicos randomizados.

Nível de evidência $\mathbf{B}$ : dados obtidos de umúnico ensaio clínico randomizado ou de vários estudos não randomizados.

Nível de evidência C: dados obtidos de opiniões consensuais de especialistas no assunto.

\section{Dissecções Agudas da Aorta}

\section{Dissecções agudas tipo A}

Nas dissecções envolvendo a aorta ascendente, a intervenção cirúrgica deve ser imediata, e tem por objetivos evitar ruptura e morte por tamponamento cardíaco, corrigir a regurgitação aórtica quando presente, evitar a isquemia miocárdica, excluir o local de laceração da íntima, e redirecionar o fluxo pela luz verdadeira aos ramos supra-aórticos e à aorta descendente ${ }^{1-7}$.

$\mathrm{Na}$ escolha da técnica de reconstrução cirúrgica, três questões devem ser consideradas: $1^{\circ}$ ) o diâmetro e o estado da raiz da aorta e dos seios de Valsalva no momento da intervenção e, se possível, previamente ao evento agudo; $2^{\circ}$ ) as condições da válvula aórtica; e $3^{\circ}$ ) a extensão ou mesmo presença de lesão da íntima no arco transverso.

Se os diâmetros da aorta ascendente e da raiz da aorta são normais, e não há desalinhamento do plano comissural da válvula aórtica nem distorção dos óstios coronarianos, a correção, usualmente, envolve a interposição de um enxerto reto de dacron, anastomosado proximalmente na porção sinotubular da aorta. Se existe perda de sustentação de uma ou mais comissuras da válvula aórtica, a mesma deverá ser ressuspendida a partir do reparo dos ângulos comissurais, antes da inserção do enxerto reto. Entretanto, se não há possibilidade de correção da insuficiência aórtica pela valvoplastia ou se a válvula aórtica é bicúspide, deve-se proceder à substituição por uma prótese, antes do implante supracomissural do enxerto ${ }^{6-8}$, embora alguns autores relatem sucesso com o reparo de válvulas bicúspides ${ }^{9}$.

A abordagem convencional para correção cirúrgica da dissecção tipo A é a esternotomia mediana, com o circuito de circulação extracorpórea (CEC) estabelecido a partir da canulação do átrio direito (AD) e da artéria femoral, utilizando-se geralmente hipotermia moderada de $28^{\circ} \mathrm{C}$ a $32^{\circ} \mathrm{C}$.

Quando a DA ocorre em uma aorta ascendente previamente dilatada, ou em pacientes com ectasia ânulo-aórtica, com ou sem síndrome de Marfan, a correção cirúrgica, necessariamente, requer a substituição da válvula aórtica, da porção sinotubular e dos seios de Valsalva, e reimplante dos óstios coronarianos, utilizando-se um enxerto composto por prótese valvar e malha de dacron, conhecido como tubo valvado ${ }^{10,11}$.

$\mathrm{Na}$ técnica originalmente descrita por Bentall e De Bono $^{12}$, os óstios coronarianos são incluídos na porção as- 
cendente do tubo de dacron, através de anastomoses diretas, realizadas após o implante valvar. A significativa incidência de pseudo-aneurismas tardios, associada à dificuldade do reimplante direto das coronárias, em casos de dilatação não muito importante dos seios de Valsalva, sustentam a modificação proposta por Kouchoukos e cols., ${ }^{13}$ na qual os óstios coronarianos são excisados e implantados no tubo sob a forma de botões; esta técnica, denominada button Bentall, tem apresentado baixa mortalidade hospitalar e menor probabilidade de eventos tardios ${ }^{14-16}$. Alternativamente, a modificação proposta por Cabrol e cols. ${ }^{17} \mathrm{em}$ que um segmento de PTFE é conectado à porção ascendente do tubo de dacron, anastomosada aos óstios coronarianos, de modo término-terminal, pode ser útil em pacientes idosos, ou em casos em que há necessidade de reconstrução muito complexa dos demais segmentos da aorta torácica.

A utilização de auto-enxerto ou homoenxerto de válvula pulmonar para reconstrução da raiz da aorta, embora preconizada por alguns autores ${ }^{18-21}$, tem apresentado elevada incidência de reoperações por degeneração tardia ${ }^{22}$, e deve ser reservada a casos selecionados, especialmente em pacientes idosos ou com endocardite associada ${ }^{23}$.

Recentemente, técnicas de preservação e remodelamento da válvula aórtica e da raiz da aorta têm sido sugeridas por autores como David e Feinde ${ }^{24}$ e Sarsam e Yacoub ${ }^{11}$. Nelas, os óstios coronarianos são excisados e a porção sinotubular é recortada entre 3 e $5 \mathrm{~mm}$ acima do anel, de forma a manter as cúspides e a linha de inserção dos ângulos comissurais intactas; uma sutura de pontos separados com pledgets de teflon reforça a junção ânulo-aórtica remanescente, que é anastomosada ao enxerto de dacron; por fim, os botões coronarianos são reimplantados de forma similar à técnica de button Bentall. Como as técnicas de remodelamento são mais complexas e demandam em geral mais tempo do que as que utilizam o tubo valvado, devem ser empregadas por cirurgiões com boa experiência em situações eletivas, permanecendo os procedimentos que utilizam o enxerto composto como recomendáveis na ectasia ânuloaórtica ${ }^{23}$. Quando eventualmente a delaminação da parede aórtica atinge os óstios coronarianos de forma a impedir uma reconstrução satisfatória, a alternativa se restringe ao(s) enxerto(s) de veia(s) safena(s).

Nas dissecções tipo A, para melhor hemostasia das suturas proximal e distal, podem ser utilizadas lâminas de teflon, adesivos teciduais, como a cola de gelatina-resorcina-formaldeído (GRF), ou ambos; tradicionalmente, os folhetos separados pela falsa luz são unidos por uma linha de sutura que inclui uma lâmina de teflon, interposta entre os folhetos, ou colocada como um reforço externo. Embora não haja consenso quanto ao benefício dos adesivos biológicos, e este produto não seja aprovado para uso nos EUA, a GRF é preconizada por grande parte dos autores, como adjuvante ${ }^{23}$, ou mesmo isoladamente ${ }^{25}$.

Não obstante os excelentes resultados cirúrgicos de alguns grupos, que relatam uma mortalidade hospitalar entre 6 e $12 \%{ }^{3,4,6,7,16,25-28}$, o International Registry of Acute
Aortic Dissection ${ }^{29}$, em levantamento retrospectivo de 464 casos de dissecções agudas da aorta atendidos em 12 centros de referência nos EUA, entre 1996 e 1998, aponta uma mortalidade operatória de $26 \%$ na dissecção aguda tipo A, valor possivelmente mais aproximado da média em nossa realidade.

Quando a dissecção aórtica envolve o arco transverso, a discussão nas diversas abordagens propostas em geralé centrada $\mathrm{em} 1^{\circ}$ ) quando incluir o arco no reparo cirúrgico, $2^{\circ}$ ) de que forma e em que extensão reconstruí-lo, e $3^{\circ}$ ) qual o melhor método de proteção cerebral a ser empregado.

É geralmente aceito que, quando a laceração da íntima não se encontra dentro do arco transverso, o mesmo pode ser reparado através de uma abordagem aberta (sem pinçamento), unindo os folhetos da parede aórtica, anastomosando ao enxerto da aorta ascendente, e redirecionando o fluxo à luz verdadeira, técnica descrita comohemiarch repair $^{30-36}$. Entretanto, em cerca de 10 a $20 \%$ das DA, a lesão intimal ocorre dentro da porção transversa do arco, o que torna indispensável a reconstrução completa e o reimplante dos troncos supra-aórticos, em bloco ou separadamente ${ }^{36}$. Esta tomada de decisão muitas vezes é difícil, na medida em que a morbidade e a mortalidade da intervenção total sobre o arco parece ser substancialmente superior aohemiarch repair, pelo maior tempo necessário de parada cardiocirculatória (PCC) e maior nível de hipotermia, que se traduz em maior incidência de dano cerebral permanente e sangramento por discrasia.

Em uma série histórica de 60 casos de DA envolvendo o arco, operados noTexas Heart Institute entre 1976 e 1982, e com uso de PCC hipotérmica, Livesay e cols. ${ }^{37}$ relataram taxas de reintervenção por hemorragia e de AVE pós-operatório de $19 \%$ e $10 \%$, respectivamente.

Na experiência recente do grupo doMount Sinai Medical Center, a substituição total do arco transverso foi empregada em 11 de 19 casos de DA tipo A, com um tempo médio de PCC sob hipotermia profunda de $56 \mathrm{~min}$, e com ocorrência de sangramentos excessivos (que justificaram o uso de derivação periaórtica para o átrio direito) em $40 \%$ dos pacientes $^{38}$.

Borst e cols. ${ }^{34}$, utilizando as técnicas de hemiarch repair ou de reconstrução total do arco, em 92 pacientes com DA, demonstraram que o tempo médio de PCC duplicou e a mortalidade triplicou na segunda abordagem $(17 \mathrm{~min}$. vs 34min., e $12 \%$ vs 36\%, respectivamente). Similarmente, Crawford e cols. ${ }^{33}$, estudando 82 pacientes com DA tipo A, relataram mortalidade significativamente maior na troca completa do arco $(31 \%)$, do que na intervenção restrita à aorta ascendente ou ao reparo parcial do $\operatorname{arco}(17 \%)$.

Esses achados, assim como outros relatos ${ }^{30,31,39,40}, \mathrm{pa}-$ recem substanciar uma abordagem cautelosa sobre o arco, com preferência à técnica dohemiarch repair com anastomose aberta, e uso adjuvante de adesivo biológico, à exceção de casos com grande destruição intra-arco ou descontinuidade com a porção descendente da aorta torácica; nesta situação, a melhor alternativa passa a ser a inserção um 
enxerto tubular de dacron na aorta descendente, cuja extremidade distal é desprovida de anastomose ("tromba de elefante"), e reimplante dos ramos supra-aórticos ${ }^{41,42}$. Na eventualidade futura de indicação de correção da aorta descendente, a extremidade livre do enxerto pode ser anastomosada diretamente à parede aórtica, ou estendida por meio de um tubo adicional a segmentos mais distais, através de toracotomia esquerda.

Entre os métodos descritos para proteção cerebral, durante intervenções no arco transverso, os mais utilizados têm sido $1^{\circ}$ ) a PCC sob hipotermia profunda, $2^{\circ}$ ) a PCC hipotérmica com perfusão cerebral retrógrada, e $3^{\circ}$ ) a $\mathrm{PCC}$ hipotérmica com perfusão cerebral anterógrada, pela artéria carótida ou, mais recentemente, pelo eixo subclávio-axilar.

Ainda nos anos 70 e 80, a PCC hipotérmica consolidou-se como método preferencial de proteção cerebral, por facilitar a inspeção do arco quanto à presença de lesão intimal, permitir a atuação em toda sua extensão, incluindo a porção proximal da aorta descendente, e impedir que o pinçamento da aorta junto ao arco pudesse gerar sítios de dissecção junto à anastomose distal ${ }^{30,37,43,44}$. A técnica consiste no estabelecimento da CEC pela canulação ADfemoral, no resfriamento lento até 18 a $20^{\circ} \mathrm{C}$ (temperatura em que a taxa metabólica é de 18\% da normal) e no uso de bolsas de gelo para resfriamento tópico da cabeça e pescoço. Oreaquecimento também deve ser lento, cerca de $1^{\circ} \mathrm{Cacada}$ 3 min., para minimizar a hemólise e os efeitos deletérios sobre os fatores de coagulação. Diversos grupos têm apontado eficácia e segurança da PCC hipotérmica, quanto à morbidade neurológica, se o período de PCC for de até $45 \mathrm{~min}$, com uma taxa de AVE entre $3 \%$ e $12 \%{ }^{34,37,45}$, embora autores como Ergin e cols ${ }^{44,46}$ relatem resultados favoráveis com até 60 min de PCC e sugiram que o dano neurológico está mais relacionado à idade avançada e à doença aterosclerótica cerebral previamente existente, do que à PCC em si. Entretanto, dados consistentes de Svensson e cols. ${ }^{47}$, na análise de 656 casos de PCC hipotérmica, apontam aumento significativo da taxa de AVE pós-operatório quando o tempo excede a 40min, e da mortalidade hospitalar com PCC superior a 60min. Recentemente, tem sido proposta a monitorização da taxa metabólica cerebral, pela aferição da saturação de oxigênio no bulbo jugular, como parâmetro para iniciar o período de PCC hipotérmica, mas sua aplicabilidade e segurança necessitam ainda ser comprovadas ${ }^{48}$.

A retroperfusão cerebral (RPC) pela veia cava superior durante a PCC, introduzida por Ueda e cols. ${ }^{49}$, tem as vantagens de proporcionar um resfriamento cerebral sustentado, lavar metabólitos resultantes da isquemia, retirar o ar ou mesmo debris com potencial emboligênico, e permitir a infusão de substratos nutricionais durante a PCC. Entretanto, potenciais limitações como distribuição intracerebral não homogênea, presença de válvulas no sistema jugular ${ }^{50}$, edema cerebral $^{51}$, fluxo cerebral insuficiente ${ }^{52}$, e sangramento no campo operatório, têm tornado seu emprego discutível.

Coselli e cols., ${ }^{53}$ em estudo recente de 479 casos de reconstrução do arco aórtico, em que utilizaram a PCC hipotérmica com RPC em 290 (60\%), e PCC hipotérmica isolada em
189(40\%), demonstraram uma mortalidade hospitalar significativamente menor no grupo submetido à RPC $(3,4 \% \mathrm{vs}$ $6,3 \%$ ). Quanto à eficácia da proteção cerebral, achados favoráveis à RPC também foram descritos por Safi e cols. ${ }^{54}$,em levantamento retrospectivo de 161 pacientes submetidos à cirurgia do arco: o efeito protetor contra derrame cerebral foi três vezes superior quando a RPC foi empregada ( $3 \%$ vs $9 \%$ ), e este benefício foi maior em pacientes $>70$ anos. No entanto, não houve diferença significativa na taxa de AVE ou óbito entre os grupos, quando o tempo de PCC foi inferior a 60 min, o que pode inferir que o benefício da RPC possa ter ocorrido, na realidade, somente pelo uso da hipotermia profunda.

Em estudo multicêntrico japonês, coordenado por Ueda e cols. ${ }^{49}$, foram analisados os resultados de 249 casos de substituição do arco aórtico utilizando RPC, entre 1994 e 1996: o tempo médio de RPC foi de 46 min, a taxa de AVE pós-operatório foi de 4\%, e a mortalidade hospitalar 13\%. A análise multivariada identificou o tempo prolongado de CEC, a idade avançada e a urgência da cirurgia, como os mais importantes fatores de risco relacionados aos desfechos AVE ou óbito.

Outra modalidade de proteção cerebral inclui a perfusão anterógrada durante a PCC, podendo-se utilizar a infusão de sangue gelado pelas artérias supra-aórticas (cerebroplegia), ou a manutenção da perfusão pela artéria axilar, com pinçamento do tronco braquiocefálico. Na técnica originalmente descrita por Guilmet e cols. ${ }^{55}$, a temperatura do paciente é mantida em $25^{\circ} \mathrm{C}$ durante a PCC, parte do sangue do oxigenador é resfriado entre 6 e $12^{\circ} \mathrm{C}$, e infundido diretamente nas carótidas a um fluxo de 200 a $250 \mathrm{ml} / \mathrm{min}$., e com uma pressão de 60 a 70 mmHg. Entre 1984e 1998, os autores utilizaramPCC e cerebroplegia em 171 casos de substituição do arco aórtico, 42 dos quais devido a dissecções tipo A; o tempo médio de CEC foi de $121 \mathrm{~min}$, o tempo médio de perfusão cerebral seletiva $60 \mathrm{~min}$, a mortalidade hospitalar $17 \%$, e a ocorrência de dano neurológico pós-operatório $13 \%{ }^{56}$.Embora outros estudos também sugiram superioridade da perfusão anterógrada sobre a RPC e a PCC hipotérmica, tanto em nível clínico ${ }^{51,57}$ como experimental ${ }^{52}$, complicações como sangramento nos sítios de canulação e hemorragia intracerebral têm restringido seu uso a poucos centros.

A canulação da artéria axilar, e a manutenção do fluxo por uma das carótidas durante a PCC, descrita por Sabik e cols.$^{58}$, têm as vantagens de evitar a manipulação da artéria femoral, freqüentemente acometida pela dissecção, de manter sempre o fluxo no sentido anterógrado pela luz verdadeira (importante quando se utiliza a tromba de elefante), e de não necessitar de hipotermia profunda, o que minimiza as complicações discrásicas. Além disso, eliminam-se os problemas de má perfusão de órgãos nobres, e de criação de novos pontos de reentrada, pelo aumento de pressão na falsa luz, como bem demonstrado por Van Arsdell e cols. ${ }^{59}$. Nesta técnica, a artéria axilar é dissecada no nível do sulco delto-peitoral, preferencialmente à direita, a CEC mantida via artéria axilar-átrio D, e a temperatura levada a aproximada- 
mente $24^{\circ} \mathrm{C}$; estabelecida a PCC, a artéria inominada é pinçada na sua origem, e o fluxo baixado para 150 a $300 \mathrm{ml} /$ min, para reconstrução do arco.

A experiência inicial tem sido positiva, em pequenas séries ${ }^{60,61}$, mas sua eficácia e segurança ainda necessitam ser comprovadas.

As recomendações para o tratamento cirúrgico das dissecções agudas do tipo A encontram-se listadas na tabela I.

\section{Dissecções agudas tipo B}

Ainda existe concordância geral de que o tratamento cirúrgico das dissecções agudas tipo B seja condicionado à presença de complicações, como sinais de ruptura aórtica (hemotórax, expansão rápida do diâmetro aórtico, alargamento de mediastino), formação de pseudoaneurisma, isquemia grave visceral ou de extremidades, ou progressão da dissecção durante terapia medicamentosa, caracterizada por dor persistente ou recorrente. Também casos de aorta previamente aneurismática que sofrem dissecção aguda devem ser considerados para cirurgia de urgência.

Entretanto, grupos como a Stanford University demonstraram, em uma coorte de 136 pacientes com DA tipo $\mathrm{B}$, que pacientes jovens e de baixo risco operatório podem ser direcionados para uma abordagem mais agressiva, com cirurgia precoce, sem aumento de mortalidade hospitalar (11\%), quando comparados à terapia medicamentosa, o que evitaria a degeneração aneurismática crônica da aorta descendente $^{62}$. Ainda que extremamente favoráveis, estes resultados não foram considerados reprodutíveis por outros centros. De fato, o International Registry of Acute Aortic Dissection $^{29}$, que pode ser considerado uma expressão do mundo real, revela que, nos casos de dissecções tipo B tratados conservadoramente, a mortalidade em 30 dias foi de apenas $10 \%$, enquanto nos pacientes operados a mortalidade foi $31 \%$ e a incidência de paraplegia $18 \%$.

A abordagem cirúrgica nas DA de aorta descendente, habitualmente, consiste na substituição da porção afetada por um enxerto tubular de dacron, através de toracotomia esquerda no $4^{\circ} \mathrm{e} / \mathrm{ou} 7^{\circ}$ espaço intercostal, o que pode ser realizado com clampeamento simples proximal ${ }^{63,64}$, com CEC átrio-femoral com ou sem hipotermia ${ }^{65-68}$, CEC parcial fêmuro-femoral ${ }^{69}$, ou até mesmo com uso da PCC sob hipotermia profunda para uma anastomose proximal aberta ${ }^{70}$.

Outra técnica referida para correção das dissecções do tipo B é a "tromba de elefante", inicialmente proposta por Borst e cols. ${ }^{71}$ para o tratamento cirúrgico estagiado de aneurismas complexos. Após esternotomia mediana e CEC convencional, uma PCC hipotérmica é induzida, e o arco transverso aberto longitudinalmente; um enxerto tubular de dacron é introduzido na porção proximal da aorta descendente e anastomosado apenas no segmento proximal, após a subclávia esquerda, ficando a extremidade distal livre na aorta torácica. Posteriormente, Buffolo e cols. ${ }^{72}$ estenderam a aplicação dessa técnica a todos os casos de dissecções agudas do tipo B, a despeito da presença de complicações; os autores realizaram 70 inserções consecutivas da "tromba de elefante", entre 1988 e 1995, por esternotomia mediana e curto período de PCC hipotérmica (média 31 min), e apresentaram mortalidade hospitalar de $20 \%$ e uma curva de sobrevida em 5 anos de $62 \%$.

Para o manejo da isquemia renal, mesentérica ou de extremidades, principalmente quando a origem destes vasos encontra-se na falsa luz ${ }^{73}$, é geralmente aceito que a fenestração por cateter deva ser o método de escolha, o que é justificado pela elevada mortalidade que acompanha a cirurgia das DA tipo B nessas situações: 50 a $70 \%$ na isquemia renal $^{74,75}, 89 \%$ na isquemia mesentérica ${ }^{74,76-78}$ e até $87 \%$ na isquemia periférica ${ }^{75,77}$.

Desde que o primeiro sucesso com a fenestração por cateter balão, para comunicar a falsa com a verdadeira luz, em um caso de DA tipo B com isquemia mesentérica, foi descrito por Williams e cols. ${ }^{79} \mathrm{em} 1990$, vários estudos têm procurado validar esta abordagem ${ }^{80-87}$, também o advento dos stents aórticos, originalmente utilizados para exclusão dos aneurismas abdominais infra-renais, introduz uma nova alternativa do manejo das dissecções complicadas de aorta descendente.

Em geral, ramos comprometidos por obstrução estática da sua origem são mais bem tratados pelo implante intraluminal de stents, enquanto vasos obstruídos pelo des-

\begin{tabular}{|c|c|c|}
\hline Recomendações & Classe de evidência & Nível de evidência \\
\hline 1. Cirurgia imediata para evitar ruptura / tamponamento / morte & I & $\mathrm{C}$ \\
\hline 2. Enxerto reto na aorta ascendente, se raiz da aorta e válvula aórtica normais & I & $\mathrm{C}$ \\
\hline $\begin{array}{l}\text { 3. Enxerto reto na aorta ascendente e ressuspensão valvar aórtica, se raiz da aorta normal e válvula insuficiente } \\
\text { por perda de sustentação }\end{array}$ & I & $\mathrm{C}$ \\
\hline 4. Tubo valvado, se aorta ascendente dilatada ou ectasia ânulo/aórtica e válvula aórtica insuficiente & I & $\mathrm{C}$ \\
\hline 5. Auto ou homoenxerto, se (situação $n^{\circ}$ 4) associada à endocardite & IIA & $\mathrm{C}$ \\
\hline 6. Ressuspensão valvar aórtica e remodelamento da raiz da aorta em síndrome de Marfan & IIA & $\mathrm{C}$ \\
\hline $\begin{array}{l}\text { 7. Reparo parcial do arco aórtico (hemiaroh repair), se dissecção compromete o arco, mas não há destruição ou } \\
\text { lesão da íntima }\end{array}$ & I & $\mathrm{C}$ \\
\hline 8. Reconstrução total do arco, se há destruição ou lesão da íntima dentro do mesmo & I & $\mathrm{C}$ \\
\hline $\begin{array}{l}\text { 9. Em caso de intervenção no arco, reconstrução aberta com método de proteção cerebral (PCC hipotérmica - } \\
\text { retroperfusão cerebral - cerebroplegia - perfusão axilar) }\end{array}$ & I & $\mathrm{C}$ \\
\hline $\begin{array}{l}\text { 10. Enxerto(s) de veia(s) safena(s), se óstios coronarianos comprometidos pela delaminação e não passíveis } \\
\text { de reimplante }\end{array}$ & & \\
\hline
\end{tabular}


locamento dinâmico da coluna de sangue da falsa luz podem ser reperfundidos pela fenestração com cateter balão, com ou sem implante de stent na luz verdadeira. Em outras situações, stents ${ }^{86}$ podem ser empregados na luz verdadeira para manter a posição de origem de alguns ramos, ou para manter aberta uma fenestração ${ }^{88}$. Outra indicação da fenestração endovascular pode ser a promoção de uma reentrada em casos em que a falsa luz em fundo cego comprime significativamente a verdadeira, ainda que esta manobra possa aumentar o risco embolização periférica ${ }^{88,89}$, ou de dilatação aneurismática em longo prazo, por manter a falsa luz patente ${ }^{86,90}$.

O objetivo técnico da fenestração é criar uma "janela" entre falsa e verdadeira luz, através da "rasgadura" da camada íntima, habitualmente junto à artéria a ser salva; de preferência, o sentido deve ser da luz menor (em geral a verdadeira) em direção à luz maior (geralmente a falsa), utilizando uma agulha especial ${ }^{73,82,87,88}$ e, se possível, ultra-sonografia intravascular (IVUS), seguida de um cateter balão de 12 a $15 \mathrm{~mm}$, que é insuflado para promover ampliação transversa da comunicação ${ }^{83}$. Se necessário, um stent de 10 a $14 \mathrm{~mm}$ pode ser implantado para impedir a oclusão da fenestração, por mecanismo de flap ou por trombose ${ }^{88}$.

Os resultados com a reperfusão de ramos obstruídos pela dissecção aórtica, através da fenestração percutânea, mesmo que provenientes de estudos não controlados ${ }^{86-88,91}$, têm sido consistentes: a taxa de restauração do fluxo varia entre 90 e $100 \%$, a média de mortalidade em 30 dias é de $10 \%$,e nenhum procedimento adicional de revascularização foi necessário, em um seguimento médio de 12 meses. Além disso, todos os óbitos relacionaram-se à irreversibilidade da isquemia no momento da intervenção, à progressão da dissecção ou a complicações cirúrgicas de intervenções combinadas.

O implante de stents na aorta descendente envolve uma diversidade maior de dispositivos e técnicas, sendo especialmente importante a margem de aorta sadia em relação à origem da artéria subclávia esquerda, a extensão da dissecção, a presença de reentrada(s) e o envolvimento de troncos viscerais. Habitualmente, o diâmetro da endoprótese, entre 2 e $4 \mathrm{~cm}$, é estimado por ressonância magnética, tomografia computadorizada, ecocardiografia transesofágica ou pelo IVUS, sendo variável o comprimento ou mesmo o uso de mais de um dispositivo, de acordo com a extensão da lesão. Dependendo da proximidade dos ramos viscerais, uma parte da prótese pode ser não recoberta, para manter a patência dos óstios envolvidos. A artéria femoral direita é o local mais comum de inserção; nela o posicionamento da endoprótese é guiado por angiografia, IVUS ou ambos os métodos. A monitorização da pressão arterial é criteriosa durante o implante, tendendo a elevar-se durante a expansão dos dispositivos e a cair significativamente após sua colocação. Recomenda-se que a PAM seja mantida entre 50 e $60 \mathrm{mmHg}$, o que pode requerer o uso de nitroprussiato de sódio $^{23}$. Ainda que a maioria dos stents disponíveis seja auto-expansível, um balão pode ser útil para melhor fixação e modelamento da luz verdadeira.
Resultados preliminares indicam que o uso de stents nas dissecções complicadas tipo B é mais seguro e carreia riscos menores quando realizados por via percutânea, em relação ao implante por cirurgia aberta ${ }^{92}$, e que a incidência de paraplegia pode passar a ser significativa com o implante de próteses com extensão superior a $15 \mathrm{~cm}^{92,93}$. Por isso, diante da necessidade de tratar segmentos mais longos da aorta, nos casos com reentrada(s) distal(is), deve-se dar preferência a implantes consecutivos de próteses mais curtas, que têm a vantagem adicional de modelar melhor casos aortas que apresentam curvatura descendente mais exuberante. Também pode ser útil a realização de by-pass carotídeo-subclávio antes do implante, em casos muito próximos à emergência da subclávia esquerda, manobra que na experiência de Grabenwöger e cols. ${ }^{94}$ chega a ser empregada em cerca de $40 \%$ dos pacientes. O seguimento em curto prazo tem demonstrado que a obliteração da lesão da íntima se mantém e o diâmetro aórtico dimimui com a trombose da falsa luz; nos casos em que são descobertos vazamentos periprótese (leaks); naqueles em que se identificaram reentradas não vistas no primeiro diagnóstico, o tratamento em geral pode ser o implante de novostent $t^{95}$.

Recentemente, extraordinária contribuição tem sido proposta pelo grupo da Escola Paulista de Medicina, como uso percutâneo de stents auto-expansíveis, em todos os casos de dissecções da aorta descendente. Palma e cols. ${ }^{96}$ submeteram 70 pacientes com dissecções tipo B (das quais $60 \%$ eram dissecções verdadeiras e $40 \%$ correspondiam a hematoma intramural ou a úlceras penetrantes), ao implante por via femoral de endopróteses recobertas de poliéster (Braile Biomédica ${ }^{\circledR}$ ), sob anestesia geral, heparinização sistêmica e hipotensão induzida. O procedimento foi considerado um êxito em 65 (93\%) pacientes, através da documentação de exclusão da falsa luz à aortografia, tendo sido necessária em 5 (7\%) a conversão para cirurgia; não houve ocorrência de paraplegia ou óbito. Em um seguimento médio de 29 meses ( 1 a 55 meses), 91\% dos pacientes encontravam-se vivos, sendo que em $49 \%$ foi requerida a inserção de stents adicionais. Na atualização mais recente desta série ${ }^{97}$, com 120 casos de dissecções tipo B, os resultados mantiveram-se consistentes: a mortalidade hospitalar foi $10 \%$, a conversão cirúrgica ocorreu em 6 (5\%) casos, não houve paraplegia (ainda que $2 / 3$ da aorta descendente tenham sido excluídos em 38 casos, e a região compreendida entre T9 e T12 tratada em 34 pacientes), e a sobrevida tardia foi de $87 \%$. A necessidade de implante de mais de um stent ocorreu em $51 \%$ da amostra, em 14 pacientes a artéria subclávia esquerda foi intencionalmente ocluída pela endoprótese e, em apenas 1 caso, foi necessária a derivação cirúrgica carotídeo-subclávia ${ }^{97}$.

Em síntese, não há dúvidas sobre o benefício das técnicas intervencionistas percutâneas no manejo das complicações relativas às dissecções agudas da aorta descendente, em comparação à cirurgia convencional. Mesmo assim, questões como se o implante rotineiro de stents substituirá a abordagem inicial medicamentosa em casos não complica- 
dos, assim como o papel do implante de endopróteses na aorta descendente durante cirurgias da aorta ascendente permanecem incertas, apesar dos promissores resultados preliminares.

A tabela II sumariza as recomendações e modos de intervenção nas dissecções agudas do tipo B.

O seguimento em longo prazo dos pacientes com dissecção aguda da aorta, operados ou manejados conservadoramente na fase aguda, demonstra que a falsa luz se mantém patente em cerca de $80 \%$ dos casos ${ }^{98}$; adicionalmente, sabe-se que, em somente $10 \%$ dos pacientes operados por dissecção tipo I de De Bakey, a falsa luz mantém-se oblitera$\mathrm{da}^{99-102}$. Entretanto, essa patência não deve ser entendida como iminência de eventos catastróficos; muitas vezes ela pode ser a principal fonte de suprimento sanguíneo de alguns órgãos nobres ${ }^{98}$. Na experiência doMount Sinai MedicalCenter ${ }^{103}$, a persistência da falsa luz esteve associada à baixa incidência de degeneração aneurismática, e nenhuma diferença significativa na sobrevida tardia: dois de 18 casos de falsa luz patente necessitaram intervenção (11\%), e a curva de sobrevida livre de eventos em 5 anos foi de $83 \%$ para falsa luz trombosada, e de $64 \%$ em casos com falsa luz patente ( $\mathrm{p}=\mathrm{NS})$.

A patência da falsa luz após cirurgia para DA parece estar mais relacionada à alta freqüência de reentrada (s) distal (is), do que ao sucesso da exclusão do sítio primário de lesão, o que reforça a necessidade de um seguimento com controle clínico extremamente rigoroso, especialmente da hipertensão arterial sistêmica (HAS), e método de imagem no $1^{\circ}, 3^{\circ}, 6^{\circ}$ e $12^{\circ}$ mês e, após, anualmente ${ }^{23}$.

Há concordância de que o método preferencial para mo nitorização em longo prazo e eventual indicação de intervenção deve ser a ressonância magnética nuclear (RMN), pois ela evita a exposição à radiação ou a contrastes nefrotóxicos u sados na tomografia computadorizada (TC), e é menos invasiva que o ecocardiograma transesofágico (ETE). A comparação seriada de imagens facilita a detecção precoce de aumento das dimensões da falsa luz ou de toda a aorta, além de ser possível a identificação dos ramos viscerais importantes e sua relação com a porção dilatada ${ }^{104,105}$.

Há evidências inequívocas de que o agressivo manejo da HAS com agentes inotrópicos negativos é o mais importante fator independente na prevenção da degeneração aneurismática crônica e da ruptura; embora os betabloqueadores sejam a terapia de escolha, por diminuírem a dp/dt. Em geral a associação de mais de uma droga é requerida ${ }^{23}$.Con- forme demonstrado por DeBakey e cols. ${ }^{106}$, em 527 pacientes, após quadro de dissecção aguda da aorta, aneurismas se desenvolveram em $46 \%$ dos casos com pressão arterial não controlada, mas somente em $17 \%$ dos pacientes normotensos.

De todos os casos de óbito na experiência remota da Stanford University ${ }^{39,107}$, pelo menos $15 \%$ ocorreu por ruptura aórtica, ao passo que o acompanhamento de 20 anos realizado na Baylor College of Medicine ${ }^{106}$ revelou que $30 \%$ de óbitos tardios foram devidos à ruptura de aneurismas crônicos. Na verdade, é estimado que cerca de $30 \%$ dos casos operados por dissecção aórtica aguda sofrerão degeneração aneurismática, com mais freqüência no eixo toraco-abdominal (35\%), seguido pela aorta descendente proximal (16\%) e aorta ascendente $(14 \%)^{106,108}$. Dados semelhantes são vistos na Europa, onde é relatada uma sobrevida de 70 a $85 \%$ no primeiro ano, e de $60 \%$ em dois anos, em casos de dissecção tipo B, sendo o prognóstico melhor nos casos de falsa luz não comunicante ( $80 \%$ de sobrevida em 2 anos $)^{99}$.

Os pacientes com síndrome de Marfan devem seguir um acompanhamento familiar mais estreito, no sentido de antecipação a eventos catastróficos, ou à recorrência tardia, embora não sejam raros os casos de múltiplas intervenções ${ }^{109,110}$; por isso, a recomendação para intervenção na dilatação aneurismática da aorta torácica ou na ectasia ânulo-aórtica. Nesse grupo específico, são seguidos critérios mais precoces, comentados no próximo tópico, embora o ponto definitivo de corte devesse ser definido a partir de estudos longitudinais, ainda não disponíveis, que determinassem, em indivíduos jovens com síndrome de Marfan, o diâmetro normal da raiz da aorta para essa população ${ }^{111,112}$. Também parece ser benéfica a restrição moderada da atividade física, em crianças e adultos jovens, pela potencial associação de hipertensão induzida pelo exercício e ruptura ${ }^{113}$.

As informações sobre a história natural do hematoma intramural (HIM) são limitadas e muitas vezes conflitantes na literatura. De modo geral, pode-se considerá-lo uma condição tão potencialmente catastrófica quanto a DAA e inferir que a mortalidade seja significativa - 20 a $80 \%{ }^{114-116}$, que a evolução para dissecção verdadeira deva ocorrer em 15 a $41 \%$ dos $\operatorname{casos}^{117-120}$, a ruptura em 5 a $26 \%$ dos pacientes, e a evolução para cura espontânea, de forma variável ${ }^{121,122}$.Em um levantamento prospectivo recente de 360 casos de dissecções aórticas agudas, Nienaber e cols. ${ }^{117}$ observaram que $25(12 \%)$ apresentavam falsa luz evidente, porém sem

Tabela II - Recomendações para o tratamento das dissecções agudas tipo B.

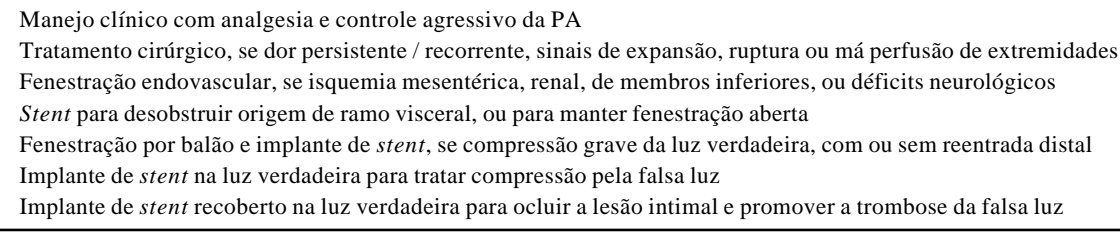

\begin{tabular}{|cl|} 
I & C \\
I & C \\
IIA & C \\
IIA & C \\
IIA & C \\
IIA & C \\
IA & B \\
\hline
\end{tabular}


lesão de íntima identificável nos exames diagnósticos. A mortalidade em 30 dias, quando havia envolvimento da aorta ascendente, foi de $80 \%$ nos casos tratados clinicamente, e zero nos pacientes operados, semelhante à DA tipo A. Por outro lado, Vilacosta e cols. ${ }^{122}$ analisaram a evolução de 21 casos de HIM, dos quais 15 eram espontâneos - 8 envolvendo a aorta ascendente e 7 confinados à porção descendente - e 6 traumáticos. No grupo considerado tipo A, 3 (37\%) casos evoluíram com morte súbita, 3 (37\%) foram operados com êxito e $2(25 \%)$ evoluíram para cura; no grupo tipo B, ocorreu $1(15 \%)$ morte súbita e $6(85 \%)$ tiveram curso clínico favorável com terapia medicamentosa, ao passo que no grupo traumático, houve cura espontânea em 3 (50\%) pacientes e evolução para óbito nos outros $3(50 \%)$, mas por causas não relacionadas à ruptura. Ainda que a amostra seja pequena, os autores sugerem que a história natural dos HIM seja melhor nos casos traumáticos ou restritos à aorta descendente, em comparação ao comprometimento da aorta ascendente.

Também a evolução da placa aórtica ulcerada é pouco conhecida e controversa nos poucos relatos disponíveis. Enquanto alguns autores a consideram tão ameaçadora quanto a dissecção aguda verdadeira, com alto potencial de ruptura, outros apontam um curso clínico benigno que dispensa tratamento cirúrgico imediato ${ }^{123,124}$. Entretanto, a presença de hematoma subintimal ou de úlceras penetrantes deve ser vista como iminência de ruptura. Em levantamento retrospectivo de 198 casos de dissecção aórtica, o grupo da Yale University ${ }^{125}$ detectou a presença de 15 úlceras penetrantes $(8 \%)$, identificadas em exames de imagem prévios ao evento agudo, das quais $13(87 \%)$ localizavam-se na aorta descendente. A idade média e as dimensões da aorta neste grupo foram significativamente superiores à amostra total, e a associação com aneurismas de aorta abdominal infrarrenal presente em $40 \%$ desses pacientes, tendo-se demonstrado na evolução hospitalar a ocorrência de ruptura em cerca de $40 \%$ dos casos. O desenvolvimento de pseudoaneurisma nessas placas ulceradas é outra complicação observada em longo prazo ${ }^{126,127}$, sendo que o desenvolvimento de dissecções verdadeiras é estimado em 10 a $20 \%{ }^{23}$.

As recomendações para acompanhamento em longo prazo de pacientes após episódio de dissecção aórtica aguda encontram-se na tabela III.

\section{Dissecções Crônicas - Aneurismas Torácicos}

\section{Aorta acendente - arco aórtico}

A aorta é considerada patologicamente dilatada quando seu diâmetro excede o considerado normal para aquela idade e superfície corporal. Quando o diâmetro supera 50\% do previsto naquele segmento analisado, configura-se o aneurisma.

$\mathrm{Na}$ aorta ascendente, a dilatação progressiva pode levar à insuficiência valvar aórtica, (mesmo em válvulas anatomicamente normais), à dissecção aguda ou à ruptura espontânea. São eventos que alteram dramaticamente a história natural e a curva de sobrevida e neles a magnitude do risco está relacionada ao diâmetro e ao tipo de doença estrutural da parede aórtica.

Indicações de substituição cirúrgica da aorta ascendente em pacientes com síndrome de Marfan, dissecções agudas, hematoma intramural e endocardite com destruição anular são suportadas em evidências consistentes. Entretanto, o momento de intervenção em pacientes assintomáticos com dilatação de origem degenerativa, assim como na associação de dilatação aórtica com válvula aórtica bicúspide, permanecem incertos.

Na síndrome de Marfan, existe concordância de que a correção cirúrgica profilática esteja indicada quando o diâmetro atinge $5,5 \mathrm{~cm}$, embora este valor possa ser menor $(4,5 \mathrm{a}$ $5 \mathrm{~cm}$ ) em pacientes com história familiar de dissecção, ruptura ou morte súbita ${ }^{23,111,128-132}$. Nos demais casos, embora a presença de sintomas ou a severidade da insuficiência aórtica possa indicar intervenção independente das dimensões da aorta, é aceito geralmente o diâmetro de $6 \mathrm{~cm}$ como indicativo de cirurgia em aneurismas assintomáticos ${ }^{23,133}$. Mesmo assim, grupos como o Mount Sinai Medical Center $^{16}$, baseados em sua experiência recente, têm proposto critérios diferenciados para intervenção, como diâmetros de 4,3cm em adultos < 40 anos com Marfan, de 4,8 a $5 \mathrm{~cm}$ em casos de achado ocasional, durante cirurgia cardíaca por outra causa, e de $4,5 \mathrm{~cm}$ nas cirurgias por válvula aórtica bicúspide $^{16}$.

As opções cirúrgicas de reconstrução da raiz da aorta e/ou da aorta ascendente já foram descritas anteriormente. Nos casos de aneurismas crônicos, alguns aspectos podem contribuir na escolha da técnica, conforme proposto por Ergin e cols. ${ }^{16}$ :

- idade e expectativa de vida em indivíduos muito idosos, ou de alto risco cirúrgico, a substituição valvar e a redução do calibre da aorta por uma sutura longitudinal com suporte pode ser uma boa alternativa ${ }^{134}$; igualmente, a troca valvar seguida do implante de enxerto reto de dacron, separadamente, pode ser apropriada a pacientes com limitada expectativa de vida ${ }^{135}$;

- qualidade da parede aórtica: uma aorta enfraquecida como nas dissecções agudas ou na síndrome de Marfan em geral exigem remoção de todo o tecido friável da raiz da aorta

\begin{tabular}{|c|c|c|}
\hline Recomendações & Classe de evidência & Nível de evidência \\
\hline Manejo contínuo da HAS com betabloqueadores & I & $\mathrm{C}$ \\
\hline Exame de imagem (RMN, CT, ETC) & I & $\mathrm{C}$ \\
\hline Restrição moderada da atividade física & I & $\mathrm{C}$ \\
\hline
\end{tabular}


e da porção ascendente; nesses casos, podem ser úteis as técnicas de button Bentall ${ }^{14}$ ou de remodelamento valvar ${ }^{136}$; - anatomia do conjunto válvula aórtica -seios de Vaisalva - porção sinotubular: quando existe dilatação do conjunto, implante de tubo valvado tipobutton Bentall ${ }^{14} \mathrm{e}$ Cabrol e cols., ${ }^{17}$ ou excepcionalmente auto/homoenxertos. Se a válvula é normal mas há dilatação dos seios e/ou da região sinotubular, podem ser empregadas técnicas de remodelamento valvar tipo David e cols. ${ }^{24}$.

- riscos de anticoagulação: em pacientes de risco hemorrágico considerável, as técnicas de remodelamento ou de auto/homoenxertos devem ser preferenciais;

- associação de endocardite valvar aórtica: embora não existam evidências conclusivas, a maioria dos grupos preconiza auto ou homoenxerto nessa situação.

Utilizando esta sistematização, o grupo doMount Sinai Medical Center publicou recentemente a experiência de 497 casos de reconstrução ânulo-aórtica ou da aorta ascendente, na maioria cirurgias eletivas $(n=310)$, corrigidas pela técnica de button Bentall $(\mathrm{n}=250)$. A mortalidade hospitalar global foi de $8 \%$, e de $5,5 \%$ quando excluídos pacientes operados em urgência; nos 250 casos de Bentall modificado, a mortalidade hospitalar foi de apenas $4 \%$, e a curva de sobrevida livre de reoperações foi de $79 \%$ em 5 anos, e de $62 \%$ em 8 anos, resultados que substanciam os autores a recomendarem esta técnica como preferencial ${ }^{16}$.

Em relação a aneurismas localizados no arco transverso, as indicações atualmente aceitas para ressecção cirúrgica incluem: $1^{\circ}$ ) diâmetro absoluto $>6$ ou $7 \mathrm{~cm}$, ou superior ao dobro do diâmetro esperado para aquele indivíduo; $2^{\circ}$ ) crescimento no diâmetro superior a 7 ou $10 \mathrm{~mm}$ por ano; $3^{\circ}$ ) dor ou sintomas compressivos e $4^{\circ}$ ) aneurismas saculares ${ }^{137,138}$. Pacientes com síndrome de Marfan em geral têm cirurgia indicada mais precocemente, com crescimento superior a 3 ou $5 \mathrm{~mm}$ por ano, oudiâmetro absoluto de $5 \mathrm{~cm}^{139}$. Os detalhamentos técnicos referentes aos métodos de reparo cirúrgico e de proteção cerebral descritos nas dissecções agudas envolvendo o arco transverso são aqui também aplicáveis.

\section{Aorta descendente - toraco-abdominal}

Em pacientes assintomáticos com aneurismas de aorta descendente (AAD), a identificação de preditores de dissecção, ruptura e/ou óbito vem sendo exaustivamente estudada, no sentido de estabelecer um ponto de corte seguro para indicação cirúrgica, na medida em que a mortalidade e a morbidade operatória são significativamente maiores aqui do que na cirurgia da aorta ascendente. Neste sentido, alguns complicados modelos de equações exponenciais têm sido propostos para cálculo da estimativa de eventos agudos em casos de $\mathrm{AAD}^{140,141}$. O grupo da Yale University tem realizado estudos evolutivos consistentes e simplificados ${ }^{77,133,142,143}$, apontando o diâmetro como o mais relevante fator independente de risco para complicações. Com base em um banco de dados de 721 pacientes com AAD, os autores acompanharam a evolução de 304 casos assintomáti- cos, com diâmetro mínimo de $3,5 \mathrm{~cm}$, e demonstraram claramente que a incidência de desfechos associados - ruptura, dissecção ou óbito - foi de 15,6\% para aneurismas com diâmetro ? a $6 \mathrm{~cm}$, e de apenas 3,9\% para diâmetros entre 4,0 e $4,9 \mathrm{~cm}(\mathrm{p}=0,004)$. Quando analisado isoladamente o desfecho ruptura, a razão de chances aumentou 27 vezes quando o diâmetro alcançou $6 \mathrm{~cm}$, em relação a casos com $4,0 \mathrm{~cm}$ ou menos $(\mathrm{p}=0,002)$. Além disso, a curva da sobrevida em 5 anos dos pacientes com diâmetro $>6 \mathrm{~cm}$ foi de apenas $56 \%$ em 5 anos, e de $85 \%$ para casos operados eletivamente ( $\mathrm{p}=0,003)$, sobrevida semelhante à encontrada na população normal, com mesma idade média. Esses achados, associados à mortalidade operatória de $11 \%$ apresentada pelo grupo $^{144}$, têm levado os autores a arbitrarem em $6 \mathrm{~cm}$ o parâmetro de indicação cirúrgica para $\mathrm{AAD}$, conduta considerada consensual pelos especialistas ${ }^{23}$.

A principal preocupação na correção cirúrgica dos aneurismas descendentes ou toraco-abdominais ainda é a paraplegia, com incidência relatada entre 4 e $32 \%$ nos trabalhos recentes ${ }^{145-156}$. De fato, a injúria medular continua a ser um evento devastador para paciente, família e equipe cirúrgica. Embora a paraplegia seja um fenômeno claramente multifatorial, pode-se dizer que sua ocorrência advém de uma ou mais das seguintes condições: $1^{\circ}$ ) duração e grau de isquemia medular; $2^{\circ}$ ) falha no restabelecimento do fluxo da medula espinhal após o reparo e $3^{\circ}$ ) injúria de reperfusão mediada bioquimicamente.

Para redução da isquemia medular, numerosas técnicas têm sido preconizadas, com resultados clínicos conflitantes.

Quando nenhum método de perfusão distal é utilizado durante a reconstrução, a aorta pode ser pinçada apenas proximalmente, como preconizado por Crawford e cols.${ }^{157} \mathrm{e}$ $\mathrm{Cooley}^{63}$, para impedir a elevação da pressão liquórica, baixando-se gradualmente o nível do clampeamento, à medida que os ramos intercostais principais e os ramos viscerais são incluídos na prótese. Enquanto existe relativa segurança com tempo de isquemia inferior a 30min, esta abordagem apresenta uma incidência proibitiva de paraparesia ou paraplegia com tempo maior de $60 \mathrm{~min}$. Em contraste, quando se pretende que a perfusão distal seja mantida para otimizar a nutrição das artérias intercostais e lombares, breves períodos de isquemia seqüencial podem ser realizados; nesta técnica, a CEC é estabelecida por via AE-femoral ou fêmuro-femoral, e permite a manutenção da perfusão até o nível de T5 ou T6 durante o primeiro pinçamento da aorta proximal e da subclávia esquerda. Concluída a anastomose proximal, o clampe distal é usualmente colocado logo acima do tronco celíaco, e os vasos intercostais principais incluídos no enxerto. No próximo passo, o clampe da prótese é posicionado abaixo do plano das intercostais, e o da aorta distal abaixo do plano das artérias renais, as quais são incluídas em bloco ou separadamente. Em seguida, o pinçamento aórtico é transferido para a porção infra-renal, e o reparo distal concluído. A esta abordagem de isquemia seqüencial tem sido inferida uma redução significativa das taxas de paraplegia e in suficiência renal ${ }^{62}$. 
Outros métodos de proteção distal podem incluir CEC com parada cardiocirculatória hipotérmica, ou o uso de shunts temporários, internos ou externos. Enquanto Kouchoukos e cols. ${ }^{70}$ suportados por incidência de $6,5 \%$ de paraplegia e de $10 \%$ de óbito em 30 dias, recomendam rotineiramente a CEC total com PCC sob hipotermia profunda, Crawford e cols. ${ }^{158}$ consideram esta técnica particularmente útil em aneurismas que envolvem parte do arco, ou extensamente calcificados em sua porção proximal, apresentando taxas de paraplegia e mortalidade, de $9,5 \%$ e $16 \%$, respectivamente.

Alternativamente, vários tipos de shunts temporários têm sido propostos, com a justificativa de necessitarem menor dose de heparina e de não prescindirem de hipotermia, minimizando as complicações hemorrágicas. A maior experiência na literatura foi publicada por Verdant e cols. ${ }^{159}$, que utilizaram by-pass aorto-aórtico ou aorto-femoral em 366 casos consecutivos de AAD, com uma mortalidade hospitalar de $12 \%$ e nenhum caso de paraplegia; entretanto, e curiosamente, mesmo com esses resultados altamente positivos, os autores têm atualmente preconizado a CEC átriofemoral, devido à incidência significativa de AVEe de lesões aórticas nos locais de derivação.

A drenagem de líquido céfalo-raquidiano (LCR) para diminuição da pressão intratecal constitui outra forma referida de proteção medular, amplamente testada em diversos modelos animais, associada ou não à infusão de drogas neurotrópico-negativas ${ }^{160-167}$.

Estudos preliminares realizados naMayo Clinic foram favoráveis ao efeito protetor da drenagem de LCR, mas os resultados subseqüentes não demonstraram significante benefício ${ }^{168}$. Outros grupos têm defendido a drenagem de LCR associada à infusão local de naloxona ${ }^{169}$, ou durante CEC átrio-femoral ${ }^{170}$.

Embora Archer e cols. ${ }^{169}$, em estudo não controlado, tenham sugerido que a naloxona associada à drenagem de LCR possa até mesmo dispensar o reimplante de artérias intercostais, nenhum benefício desta técnica foi evidenciado em ensaio randomizado subseqüente ${ }^{171}$, o que torna seu uso empírico.

O efeito da administração epidural de papaverina sobre a função da medula espinhal tem sido referido como promissor. Em modelos experimentais de clampeamento torácico por até $60 \mathrm{~min}$ em normotermia, a injeção intratecal de papaverina foi altamente efetiva em prevenir a paraplegia, tendo-se demonstrado através de radionuclídeos um aumento significativo do suprimento de sangue na porção anterior da medula espinhal ${ }^{163}$. Em nível clínico, a retirada de pequeno volume de LCR, seguida da aplicação intratecal de papaverina, também se mostrou potencialmente benéfica em alguns estudos prospectivos não controlados ${ }^{172,173}$,motivando ensaios clínicos em andamento.

Outros agentes farmacológicos propostos como adjuvantes na prevenção da paraplegia, incluem corticoesterói$\operatorname{des}^{174}$, lidocaína ${ }^{174}$, manitol ${ }^{175}$, sulfato de magnésio ${ }^{165}$, prostaglandinas ${ }^{176}$, alopurino $1^{177}$, flunarizina ${ }^{178}$, entre outros, mas nenhum se mostrou isoladamente efetivo de forma a sustentar seu emprego na prática clínica.

Uma recomendação consensual diz respeito à necessidade de reimplante do máximo de artérias intercostais principais, particularmente no 1/3 inferior do tórax e abdômen superior, entre T7 e L1. ${ }^{179}$. Vários estudos anatômicos já demonstraram que o suprimento sanguíneo da medula em nível torácico é predominantemente proveniente das artérias intercostais entre T4 e T12, e das lombares entre L1 e L4 ${ }^{180-182}$, e que entre T7 e L1, em mais de $90 \%$ dos indivíduos, emerge a mais importante artéria radicular, referida como artéria de Adamkiewicz ou radicular magna. Ainda que nos processos crônicos, como aneurismas ateroscleróticos, possa haver desenvolvimento de circulação colateral capaz de manter a função medular, mesmo quando há sacrifício extenso de ramos intercostais, ou que a trombose precoce de alguns patchs de intercostais seja um evento arteriograficamente documentado ${ }^{183}$, todo o esforço deve ser feito para o salvamento destes vasos. Nesse sentido, a preservação da parede posterior da aorta torácica terminal, proposta por Williams ${ }^{184}$, pode ser uma manobra válida.

Devido à diversidade de variações anatômicas no suprimento sanguíneo medular, que podem incluir desde uma artéria radicular magna bem desenvolvida e facilmente identificada, ou várias intercostais terminais calibrosas e, até mesmo, várias e numerosas arteríolas de pequeno calibre, modelos de mapeamento intra-operatório de artérias segmentares têm sido testados, com o objetivo de selecionar artérias a serem reimplantadas e de reduzir o tempo de pinçamento aórtico, como a infusão intra-operatória de hidrogênio para identificação dos ramos intercostais que efetivamente contribuem na perfusão medular ${ }^{185}$.

Similarmente, vários estudos experimentais e clíni$\cos ^{48,158,163,186-193}$ têm sido desenvolvidos tentando definir o papel dos potenciais evocados motores ou somato-sensoriais, na monitorização da atividade da medula segmentar e na prevenção da paraplegia, embora com resultados muito variáveis, e de recomendação ainda não consensual, quanto à eficácia como método isolado na prevenção da isquemia medular ${ }^{179}$.

Como referido no item sobre dissecções agudas, uma abordagem inovadora e altamente promissora no tratamento dos AAD é o implante de stents auto-expansíveis, introduzido pelo grupo da Stanford University ${ }^{194}$. Preliminarmente, os autores procederam ao implante percutâneo por via femoral, destents auto-expansíveis recobertos por dacron, em 13 casos de aneurismas torácicos crônicos, com diâmetro médio de $6,1 \mathrm{~cm}$. A exclusão imediata foi observada em 12 pacientes, e não houve óbito ou paraplegia na fase hospitalar, nem no seguimento médio de 11,6 meses. Em ensaio clínico desenvolvido subseqüentemente ${ }^{195}$, os mesmos autores ampliaram a experiência para 103 implantes, a maioria dos quais com risco cirúrgico proibitivo, e obtiveram sucesso imediato em $83 \%$ dos casos. A mortalidade precoce foi de $9 \%$, paraplegia foi observada em $3 \%$ dos pacientes, e $7 \%$ desenvolveram acidentes isquêmicos cerebrais durante a 
internação. O seguimento tardio de 3,7 anos demonstrou uma sobrevida livre de eventos de $53 \%$, e o grupo passou a recomendar esta técnica a pacientes selecionados de alto risco operatório. Entretanto, a maior experiência com a exclusão percutânea de AAD foi recentemente publicada por Buffolo e cols. ${ }^{97}$, que entre $1996 \mathrm{e} 2002$, procederam a $191 \mathrm{im}$ plantes de stents, em casos de dissecção tipo B $(\mathrm{n}=120)$, aneurismas torácicos verdadeiros ( $\mathrm{n}=61)$, hematomas ou úlceras penetrantes $(n=6)$ e lesão traumática da aorta descendente $(n=4)$. Em todos os pacientes, foram utilizados anestesia geral, hipotensão induzida (PAM entre 50 e $60 \mathrm{mmHg}$ ), heparinização apenas durante o procedimento (5000 UI/IV), e stents recobertos por malha de polyester (Braile Biomédica ${ }^{\circledR}$ ), cujo tamanho foi calculado como excedendo em $10 \%$ a $20 \%$ o diâmetro da aorta. A taxa de sucesso nos implantes, definida como a obliteração da laceração de íntima ou a completa exclusão dos aneurismas sem extravasamentos, foi de 91\% (174/191); a mortalidade hospitalar de $10 \%$, a conversão para cirurgia necessária em 6 (3\%) pacientes, e nenhum caso de paraplegia foi observado.

Esses resultados, confirmados em estudos subseqüentes, talvez representem o mais importante avanço no tratamento de uma das doenças cardiovasculares de mais alta morbidade e mortalidade, e mais desafiadora para os cirurgiões, que são os aneurismas torácicos e toraco-abdominais.

A tabela IV apresenta as recomendações para o manejo dos aneurismas crônicos da aorta torácica.

\section{Aorta abdominal infra-renal}

Em poucas situações na medicina, uma intervenção cirúrgica dita “profilática” tem um impacto tão grande na modificação da história natural de uma doença, como nos aneurismas de aorta abdominal infra-renal (AAA), não somente por sua alta prevalência ( 90 a $95 \%$ de todos os casos de aneurismas de aorta), como também pelo incremento de morbidade e mortalidade que acompanha a correção em caráter de urgência (risco de óbito 10 vezes maior do que na cirurgia eletiva). Para o estabelecimento de diretrizes para indicações de reparo eletivo dos AAA, no sentido de substanciar uma tomada de decisão, alguns aspectos sobre comportamento desta doença devem ser considerados.

Aneurismas abdominais são encontrados incidentalmente com freqüência, sobretudo na população idosa. Vários estudos têm estimado que AAA são encontrados em
$2 \%$ dos indivíduos aos 60 anos, e em cerca de $5 \%$ das pessoas com idade $>70$ anos, sendo 2 a 3 vezes mais comum no homem do que na mulher ${ }^{196,197}$.

A associação de AAA com algumas doenças é conhecida e relativamente previsível: sabe-se que AAA podem ser encontrados em cerca de $5 \%$ dos pacientes com aterosclerose coronariana ${ }^{198}$, em $9 \%$ nos casos de arteriopatia periférica ${ }^{199}$, e em 30 a $50 \%$ dos pacientes com aneurismas poplíteos ou femorais ${ }^{200}$.

AAA são facilmente detectáveis pelo exame clínico e por métodos diagnósticos não invasivos. Em linhas gerais, um examinador experiente pode palpar diretamente aneurismas a partir de $5 \mathrm{~cm}$ de diâmetro, mas a precisão do diagnóstico somente pela palpação é $<50 \%$, ao passo que a ultrasonografia é hábil em diagnosticar AAA de qualquer diâmetro em $100 \%$ dos casos.

A ruptura dos AAA é considerada um problema de saúde pública de grande magnitude: estimam-se em 15.000 óbitos ao ano nos EUA os casos que conseguem chegar ao hospital, e talvez o dobro ou triplo deste número se incluídos todos os casos de morte súbita por AAA que ocorrem fora do ambiente hospitalar ${ }^{197}$.

A ruptura dos AAA é mais relacionada a aneurismas grandes, de crescimento rapidamente progressivo ou com início recente de sintomas. Estima-se que o risco de ruptura em 5 anos para aneurismas com menos de $5 \mathrm{~cm}$ de diâmetro seja inferior a $5 \%$, enquanto nos aneurismas maiores de $5 \mathrm{~cm}$ o risco acumulado se eleva para 25 a $43 \%{ }^{201-205}$. A análise multivariada de alguns estudos identificou, como maiores preditores de risco para ruptura, a expansão rápida documentada e a presença de dor abdominal ou lombar significante, independente do tamanho do AAA ${ }^{206-208}$.

Aneurismas pequenos crescem com velocidade variável: embora se estime que a média de expansão dos AAA deva ser de $0,4 \mathrm{~cm} /$ ano, existe uma grande variabilidade que torna impossível predizer a evolução em um indivíduo específico $^{209}$.

O risco de morte ou complicações maiores com a cirurgia eletiva dos AAA é dependente da experiência do grupo cirúrgico e do volume cirúrgico do hospital, mas na era recente a mortalidade deve ser inferior a 5\%. Embora a metanálise de estudos recentes aponte uma mortalidade média de $3,5 \%{ }^{204,210-217}$, uma variação de até $10 \%$ pode ser observada entre cirurgiões ou entre instituições ${ }^{218}$.

\begin{tabular}{|c|c|c|}
\hline Recomendações & Classe de evidência & Nível de evidência \\
\hline \multicolumn{3}{|l|}{ Aorta ascendente } \\
\hline 1. Cirurgia, se sintoma compressivo, insuficiência aórtica, ou diâmetro aórtico? $6 \mathrm{~cm}$ & I & $\mathrm{C}$ \\
\hline $\begin{array}{l}\text { 2. Em síndrome de Marfan, cirurgia profilática, se diâmetro? } 5,5 \mathrm{~cm} \text { ou ? } 5,0 \mathrm{~cm} \text { em casos } \\
\text { com história familiar de dissecção ou morte súbita. }\end{array}$ & IIA & $\mathrm{C}$ \\
\hline \multicolumn{3}{|l|}{ Aorta descendente } \\
\hline 1. Cirurgia, se sintomas ou diâmetro aórtico ? $6 \mathrm{~cm}$ & I & $\mathrm{C}$ \\
\hline 2. Implante de stents, se diâmetro aórtico ? $6,0 \mathrm{~cm}$ e anatomia favorável. & IIA & B \\
\hline
\end{tabular}


A presença de sintomas nos AAA é indicação cirúrgica consensual, independente do diâmetro, incluindo dor/ desconforto lombar ou abdominal, embolização distal ou manifestações de compressão de estruturas vizinhas. Obviamente a cirurgia de emergência é obrigatória nos casos suspeitos de ruptura.

Também aneurismas de etiologia inflamatória têm indicação de correção eletiva independente das dimensões, por se acompanharem de manifestações sistêmicas significantes, como febre e emagrecimento ${ }^{197}$.

Para pacientes assintomáticos, a indicação de intervenção deve considerar o risco de ruptura versus o risco operatório individual e a expectativa de vida. Nos anos 90, a recomendação da International Society for Vascular Surgery era de cirurgia eletiva para AAA com diâmetro? a $5,0 \mathrm{~cm}$, ou mesmo 4,0cm em pacientes com DBPOC, e baseava-se na baixa mortalidade pós-operatória encontrada nesse grupo $(1 \%)^{197,205}$. Também havia clara recomendação de contra-indicar a correção cirúrgica de AAA de qualquer diâmetro, na presença de neoplasia metastática, insuficiência cardíaca grave, ou outra condição que limitava a sobrevida em não mais de 2 a 3 anos ${ }^{197}$.

Entretanto, resultados de dois grandes ensaios clínicos recentes devem possivelmente modificar estes critérios.

No United Kingdom Small Aneurysm Trial ${ }^{219}, 1090$ pacientes com aneurismas assintomáticos com diâmetro entre 4,0 e $5,5 \mathrm{~cm}$, foram randomizados para tratamento conservador e ultra-sonografias seriadas ou reparo cirúrgico eletivo, e acompanhados por um período de 6 a 10 anos (média: 8 anos) A mortalidade operatória foi de 5,5\%, e a sobrevida em até 8 anos foi semelhante em ambos os grupos, embora ligeiramente superior no grupo que sofreu intervenção após esse período. Não foi observada diferença nesse comportamento em relação a idade, sexo ou tamanho inicial do aneurisma, e os autores concluíram que AAA assintomáticos com diâmetro ? a 5,5cm podem ser acompanhados conservadoramente, sem riscos adicionais.

Os achados também se confirmaram noAneurysm Detection and Management Veterans Affairs Cooperative Stud $y^{220}$, em que 1136 pacientes com aneurismas infra-renais com diâmetro entre 4,0 e 5,4cm foram randomizados para tratamento cirúrgico $(n=569)$ ou para controle ecográfico periódico $(n=567)$. A mortalidade hospitalar no grupo operado foi de 2,7\%, e não houve diferença na sobrevida em 5 anos entre os grupos. Não houve redução na taxa de morte relacionada a complicações do AAA no gru po com intervenção (3\% vs
$2,6 \%)$, e o risco de ruptura nos pacientes não operados foi mínimo $(0,6 \%$ por ano). Os autores não recomendam, portanto, cirurgia eletiva para AAA com até $5,4 \mathrm{~cm}$, mesmo quando a mortalidade cirúrgica do grupo for baixa.

Em contrapartida, a alta incidência de ruptura dos AAA com diâmetro $>5,5 \mathrm{~cm}$ foi bem demonstrada pelos mesmos autores ${ }^{221}$, em uma coorte de 198 pacientes com contra-indicações absolutas ao reparo cirúrgico. A taxa anual de ruptura foi de $9,4 \%$ para diâmetros entre 5,5 e $5,9 \mathrm{~cm}$; de $10,2 \%$ para casos entre 6,0 e $6,5 \mathrm{~cm}$; de $19 \%$ entre 6,6 e 7,0cm; e de $32,5 \%$ nos pacientes com diâmetro $>7,0 \mathrm{~cm}$, o que corrobora a recomendação dos estudos anteriores.

A terapia intervencionista com o implante destents tem sido recentemente proposta como alternativa ao tratamento cirúrgico dos AAA. Desde que Parodi e cols. ${ }^{222}$ realizarama primeira exclusão percutânea de um aneurisma infra-renal há 12 anos, diversos dispositivos têm sido desenvolvidos e testados, sem que haja atualmente concordância de que possa ser um eventual substitutivo da cirurgia convencional. Os resultados atuais apontam para uma taxa de sucesso imediato em torno de $95 \%$, a conversão para cirurgia tem sido necessária em cerca de 3 a $5 \%$ dos casos, e a mortalidade hospitalar relatada em aproximadamente $3 \%$. As complicações precoces mais comuns são hematomas inguinais (7\%), trombose arterial (3\%), e ruptura de artéria ilíaca $(1,5 \%)$. E longo prazo, os vazamentos (endoleaks) têm sido demonstrados em cerca de 10 a $20 \%$ dos casos, com resolução espontânea em apenas 40 a $50 \%$ deles $^{223}$. Quando a comparação entre o implante de stents e a cirurgia convencional é realizada, como nos estudos de Hallett e cols. ${ }^{224} \mathrm{e}$ de May e cols. ${ }^{225}$, pode-se observar que não existem diferenças significativas na mortalidade e morbidade precoces, pois a curva de sobrevida livre de eventos em 2 anos é marcadamente melhor no grupo cirúrgico ( $93 \%$ vs 67\%).

Em conclusão, baseado no mais recente Guidellines da American Association for Vascular Surgerye daSociety for Vascular Surgery, pode-se afirmar que $1^{\circ}$ ) pacientes assintomáticos de baixo risco devem ser considerados para cirurgia eletiva com diâmetro mínimo de $5,5 \mathrm{~cm}$ (em mulheres o diâmetro arbitrado pode ser de $5,0 \mathrm{~cm}$ ); $2^{\circ}$ ) casos de risco perioperatório significativo com $6,0 \mathrm{~cm} ; \mathrm{e} 3^{\circ}$ ) o manejo clínico ótimo deve incluir controle da hipertensão e abandono do fumo. A terapia endovascular pode ser uma alternativa válida em pacientes de alto risco cirúrgico, embora seja uma indicação não consensual226.

A tabela $V$ apresenta os critérios atuais de intervenção nos casos de AAA.

Tabela V - Critérios de intervenção nos aneurismas aórticos infrarrenais.

\begin{tabular}{|c|c|c|}
\hline Recomendações & Classe de evidência & Nível de evidência \\
\hline $\begin{array}{l}\text { 1. Tratamento cirúrgico, se sintomas - dor lombar ou abdominal, compressão de corpo vertebral ou } \\
\text { estruturas adjacentes }\end{array}$ & I & $\mathrm{C}$ \\
\hline 2. Em casos assintomáticos, cirurgia, se diâmetro ? $5,5 \mathrm{~cm}$ e baixo risco operatório / longa expectativa de vida. & I & A \\
\hline 3. Considerar diâmetro de $6 \mathrm{~cm}$, se risco operatório elevado & I & $\mathrm{C}$ \\
\hline 4. Implante de stents, se alto risco cirúrgico e anatomia favorável & IIA & $\mathrm{C}$ \\
\hline
\end{tabular}




\section{Referências}

1. Daily PO, Tueblood HW, Stinson EB, Wuerflein RD, Schumway NE. Management of acute aortic dissections. Ann Thorac Surg 1970; 10: 237-47.

2. Wolfe WG, Moran JF. The evolution of medical and surgical management of acute aortic dissection. Circulation 1997; 56 (4 pt 1): 503-5.

3. Jamieson WR, Munro Al, Miyagishima RT, Allen P, TyersFG, Gerein An. Aortic dissection: early diagnosis and surgical management are the keys to survival. Can J Surg 1982; 25: 145-9.

4. Svensson LG, Crawford ES, Hess KR, Coselli JS, Safi HJ. Dissection of the aorta and dissecting aortic aneurysms: improving early and long-term surgical results. Circulation 1990; 82 (5 suppl): IV24-IV38.

5. Pretre R, Von Segsser LK. Aortic dissection. Lancet 1997; 349: 1461-4.

6. Borst HG, Lass J, Haverich A. A new look at acute type-A dissection of the aorta. Eur J Cardiothorac Surg 1987; 1: 186-9.

7. Miller DC. Surgical Management of acute aortic dissection: new data. Semin Thorac Cardiovasc Surg 1991; 3: 225-37.

8. Culliford AT, Ayvaliotis B, Schemin R, Colvin SB, Isom OW, Spencer FC Aneurysms of the ascending aorta and transverse arch: surgical experienced in 80 patients. J Thorac Cardiovas Surg 1982; 82: 701-10.

9. Fraser CD, Wang N, Mee RB et al. Repair of insufficient bicuspid aortic valves Ann Thorac Surg 1994; 58: 386-90.

10. Gott VL, Cameron DE, Pyeritz RE et al. Composite graft repair of Marfan aneurysm of the ascending aorta: results in 150 patients. J Card Surg 1994; 9: 428-9.

11. Sarsam MA, Yacoub M. Remodeling of the aortic valve annulus. J Thorac Cardiovasc Surg 1993; 105:435-8.

12. Bental H, De Bono A. A technique for complete replacement of the ascending aorta. Thorax 1968: 23: 338-9.

13. Kouchoukos NT, Karp RB, Blackstone EH, Kirklin JW, Pacifico AD, Zorn GL. Replacement of the ascending aorta and aortic valve whit a composite graft. Results in 86 patients. Ann Surg 1980; 192: 403-13.

14. Kouchoukos NT, Marshall WG JR, Wedige-Stecher TA. Eleven-year experience with composite graft replacement o the ascending aorta and aortic valve. J Thorac Cardiovasc Surg 1986; 92: 691-705

15. Kouchoukos NT, Wareing TH, Murphy SF, Perrillo JB. Sixteen-year experience with aortic root replacement. Results in 182 operations. Ann Surg 1991;214: 308-18. Discussion318-20.

16. Ergin MA, Spielvogel D, Apaydin A, Lansman SL, McCullough JN et al: Surgical treatment of the dilated ascending aorta: when and how? Ann Thorac Surg 1999; 67: 1834-9.

17. CabrolC,Pavie A, Mesnildrey Pet al. Long-term results with total replacement of the ascending aorta and reimplatation of the coronary arteries. J Thorac Cardiovasc Surg 1986; 91: 17-25

18. Kouchoukos NT, Davila-Roman VG, Spray TL et al. Replacement of the aortic root whit a pulmonary autograft in children and young adults with aortic valve disease. NEngl J Med 1994; 330: 1-6.

19. Chambers JC, Somerville J, Stone S, Ross DN. Pulmonary autograft procedure for aortic valve disease. Circulation 1997; 96: 2206-14.

20. Oury JH, Hiro SP, Maxwell JM, Lamberti JJ, Duran CMG. The Ross Procedure: current registry results. Ann Thorac Surg 1998; 66 (suppl): S162-5.

21. Doty JR, Salazar JD, Liddicoat JR et al. Aortic Valve replacement with cryopreserved aortic allograft: ten-year experience. J Thorac Cardiovasc Surg 1998; 115: 371-80.

22. Kouchoukos NT. Aortic allografts and pulmonary autografts for replacement of the aortic valve and aortic root. Ann Thorac Surg 1999; 67: 1846-8.

23. Erbel R, Alfonso F, Boileau C, Dirsch O, Eber B et al: Task Force Report Diagnosis and management of aortic dissection. Eur Heart J 2001;22: 1673-81.

24. David TE, Feindel CM. An aortic valve-0sparing operation for patients with aortic incompetence and aneurysm of the ascending aorta. J Thorac Cardiovasc Surg 1992; 103:617-21. Discussion 622.

25. Bachet J, Gigou F, Laurian C, Bical O, Goudot B, Guilmet D. Four-year clinical experience with the gelatin-resorcine-formol biological glue in acute aortic dissection. J Thorac Cardiovasc 1982;83:212-7.

26. Najafi H, Dye S, Javid H, Hunter JA, Goldin MD, Julian OC. Acute aortic regurgitation secondary to aortic dissection. Surgical management without valve replacement. Ann Thorac Surg 1972; 14: 474-82.

27. Crawford ES, Svensson LG, Coselli JS et al. Surgical treatment of aneurysm and/ or dissection of the ascending aorta, transverse aortic arch, and ascending aorta and transverse aortic arch: Factors influencing survival in 717 patients. J Thorac Cardiovas Surg 1989; 98: 659-74

28. Galloway AC, Colvin SB, GrossiEA et al. Surgical repair of type A aortic dissection by the circulatory arrest-graft inclusion technique in sixty-six patients J Thorac Surg 1993; 105: 781-90.

29. Hagan PG, Nienaber CA, Isselbahcher EM, Bruckman D, Karavite DJ et al. The
International Registry of Acute Aortic Dissection (IRAD). JAMA 2000; 283 : 897-903.

30. Ergin MA, O`Connor J, Guinto R, Griepp RB. Experience with profound hypothermia and circulatory arrest in the treatment of aneurysms of the aortic arch. Aortic arch replacement for acute aortic arch dissections. J Thorac Cardiovasc Surg 1982; 84: 649-55.

31. Heinemann M, Laas J, Jurmann M, Karch M, Borst HG. Surgery extended into the aortic arch in acute type A dissection. Indications, techniques and results. Circulation 1991; 84: III25-30.

32. Cooley DA. Aortic aneurysm operations: Past, present, and future. Ann Thorac Surg 1999; 67: 1959-62

33. Crawford ES, Kirklin JW, Naftel DC et al. Surgery for acute dissection of ascending aorta: Should the arch be included? J Thorac Cardiovasc Surg 1992; 104:46-59.

34. Borst HG, Buhner B, Jurmann M. Tactics and techniques of aortic arch replacement. JCar Surg 1994; 9:538-47.

35. Yun KL, Glower DD, Miller DC et al. Aortic dissection resulting from tear of transverse arch: Is concomitant arch repair warranted? J Thorac Cardiovsc Surg 1991; 102:355-70.

36. Moon MR, Miller C. Aortic arch replacement for dissection. Operative Techniques in Thoracic and Cardiovascular Surgery, 1999; 4:33-57

37. Livesay JJ, Cooley DA, Reul GJetal. Resection of aortic arch aneurysms: A comparison of hypothermic techniques in 60patients. Ann Thorac Surg 1983;36: 19-28

38. Griepp RB, Ergin MA, McCullough JNet al. Use of hypotermic circulatory arrest for cerebral protection during aortic surgery. JCard Surg 1997; 12:312-21.

39. Haverich A, Miller DC, Scott WC et al. Acute and chronic aortic dissections: Determinants of long-term outcome for operative survivors. Circulation 1985 (suppl 2) 72: 22-34

40. Okita Y, Takamoto S, Ando Met al. Mortality and cerebral outcome in patients who underwent aortic arch operations using deep hypothermic circulatory arrest with retrograde cerebral perfusion: No relation of early death, stroke, and delirium to the duration of circulatory arrest. J Thorac Cardiovasc Surg 1998; 115: 129-38

41. Borst HG, Walterbusch G, Schaps D. Extensive aortic replacement using "elephant Trunk" prosthesis. Thorac Cardiovasc Surg 1983; 31:37-40.

42. Borst HG, Frank F, Schaps D. Treatment of extensive aortic aneurysms by a new multiple-stage approach. J Throac Cardiovasc Surg 1988; 95: 11-3.

43. Griepp RB, Stinson EB, Hollingsworth JF, Buehler D. Prosthetic replacement of the aortic arch. J Thorac Cardiovasc Surg, 1975; 70: 1051-63.

44. Griepp RB, Ergin A, McCullough JN et al. Use of hypothermic circulatory arrest for cerebral protection during aortic surgery. J Card Surgery 1997; 12:312-21.

45. Galloway AC, Colvin SB, LaMendola CL et al. Ten-year operative experience with 165 aneurysms of the ascending aorta and aortic arch. Circulation 1989 (supplI); 80: 249-56.

46. Ergin MA, Galla JD, Lansman SL et al. Hypothermic circulatory arrest in operations on the thoracic aorta: Determinants of operative mortality in operations on the thoracic aorta: Determinants of operative mortality and neurologic outcome. J Thorac Cardiovasc Surg 1994; 107: 788-99.

47. Svensson LG, Crawford ES, Hess KR et al: Deep hypothermia with circulatory arrest: Determinants of stroke and early mortality in 656 patients. J Thorac Cardiovasc Surg 1993;106: 19-31.

48. McCullough JN, Galla JD, Ergin A, Griepp RB. Central nervous system monitoring during operations on the thoracic aorta. Operative Techniques in Thoracic and Cardiovascular Surgery 1999; 4: 87-96.

49. Ueda Y, Miki S, Kushuhara K, Okita Y et al. Surgical treatment of aneurysm or dissection involving the ascending aorta and aortic arch, utilizing circulatory arrest and retrograde cerebral perfusion. J Cardiovasc Surg 1990; 31: 553-8.

50. Dresser LP, Mckinney WM. Anatomic and pathophysiologic studies of the human internal jugular valves. Am J Surg 1987; 154:220-4

51. YeJ, Yang J, Del Bigio MR et al. Neuronal damage after hypothermic circulatory arrest and retrograde cerebral perfusion in the pig. Ann Thorac Surg 1996; 61: 1316-22.

52. Sakurada T, Kazui T, Tanaka H, Komatsu S. Comparative experimental study of cerebral protection during aortic arch reconstruction. Ann Thorac Surg 1996; 61: 1348-54

53. Coselli JS. Retrograde cerebral perfusion. Is it valuable adjunct during circulatory arrest? In: Kawashima U, Takamoto S. Brain protection in aortic surgery. Amsterdam: Elsevier, 1997: 167-81

54. Safi H, Letsou G, Lliopoulous Det al. Impact of retrograde cerebral perfusion on ascending aortic and arch aneurysm repair. Ann Thorac Surg 1997; 63: 1601-7.

55. GuilmetD, Roux PM,B achet Jet al.Nouvelle technique de protection cérébrale: chirurgie de la crosse aortique. Presse Med 1986: 15:1096-8. 
56. Bachet J, Guilmet D, Goudot B, Dreyfus GD, Delentdecker Petal. Antegrade cerebral perfusion with cold blood: A 13-year experience. Ann Thorac Surg 1999; 67: $1874-8$.

57. Swain JA, McDonald TJ, Griffith PKet al.Low-Flow hyphotermic cardiopulmonary bypass protects the brain. J Thorac Cardiovasc Surg 1991; 102: 76-84.

58. Sabik JF, Lytle BW, McCarthy PM, Cosgrove DM. Axillary artery: an alterative site of arterial cannulation for patients with extensive aortic and peripheral vascular disease. J Thorac Cardiovasc Surg 1995; 109: 885-91.

59. Van Arsdell GS, David TE, Butany J. Autopsies in acute type A aortic dissection: Surgical implications. Circulation 1998;98 (suppl): II299-304.

60. Néri E, Massetti M, Campannini G. Carone E, Tucci E et al. Axiliary artery cannulation in type a aortic dissection operations. J Thorac Cardiovasc Surg 1999; 118:324-9.

61. Whitlark JD, Goldman SM, Sutter FP. Axillary artery cannulation in acute ascending aortic dissections. Ann Thorac Surg 2000; 69: 1127-9.

62. Miller DC. Surgical management of acute aortic dissection: New data. Semin Thorac Cardiovas Surg 1991;225-37.

63. Svensson LG, Crawford ES. Cardiovascular and Vascular Disease of the Aorta Philadelphia.PA, W.B.Saunders, 1997.

64. Scheinin AS, Cooley DA. Graft replacement of the descending thoracic aorta: Results of "open" distal anastomosis. Ann Thorac Surg 1994; 58: 19-22.

65. Von Oppel UO, Dunne T, De Groot MK, Zilla P. Traumatic aortic rupture: Twentyyearmeta-analysis of mortality and risk of paraplegia. Ann Thorac Surg 1994;58: 585-93.

66. Read RA, Moore EE, Moore FA, Haenel JB. Partial left heart bypass for thoracic aorta repair. Survival without paraplegia. Arch Surg 1993; 128: 746-50.

67. Pate JW, Fabian TC, Walker WA. Acute traumatic rupture of the aortic isthmus: repairs with cardiopulmonary byoass. Ann Thorac Surg 1995;59:90-9.

68. Chiesa R, Melissano G, Ruettimann LM, Civilini E, Tshomba Y. Surgical treatment of thoracic and thoracoabdominal aortic aneurysms: technical notes and the use of left heart bypass. J Vasc Br 2002; 1: 207-18.

69. Laschinger JC, Izumoto H, Kouchoukos NT. Evolving concepts in prevention of spinal cord injury during operations on the descending thoracic and thoracoabodminal aorta. Ann T horac Surg 1987; 44:667-74.

70. Kouchoukos NT, Daily BD, Rokkas CK et al. Hypotermic bypass and circulatory arrest for arrest for operations on the descending thoracic and thoracoabodominal aorta. Ann Thorac Surg 1995; 60: 67-77.

71. Borst HG, Frank G, Schaps D. Treatment of extensive aortic aneurysms by a new multiple stage approach. J Thorac Cardiovasc Surg 1988; 95: 11-3.

72. Palma JH, Almeida DR, Carvalho AC, Andrade JCS, BuffoloE. Surgical treatment of acute type $\mathrm{B}$ aortic dissection using an endoprosthesis(Elephant Trunk). Ann Thorac Surg 1997; 1997; 63: 1081-4.

73. Slonim SM, Nyman U, Semba CP, Miller DC, Mitchell RS, Dake MD. Aortic dissection: percutaneous management of ischaemic complications with endovascular stents and ballon fenestration. J Vasc Surg 1996; 23: 241-51. Discussion 251-3.

74. Cambria RP, Brewster DC, Gertler J et al. Vascular complications associated with spontaneous aortic dissection. J Vasc Surg 1988; 7: 199-209.

75. Laas J, Heinemann M, Schaefers HJ, Daniel W, Borst HG. Management of thoracoabodminal malperfusion in aortic dissection. Circulation 1991; 84: III20-4.

76. Walker PJ, Miller DC. Aneurysmal and ischaemic complications of type B (type III) aortic dissections. Semin Vasc Surg 1992; 5: 198-214.

77. Elefteriades JA, Hartleroad J, Gusberg RJ et al. Long-term experience with descending aortic dissection: the complication-specific approach. AnnThorac Surg 1992;53:11-20. Discussion 20-1.

78. Miller DC, Mitchell RS, Oyer PE, Stinson EB, Jamieson WS, Shumway NE. Independent determinants of operative mortality for patients with aortic dissection. Circulation 1984; 70: 153-60.

79. Williams DM, Brothers TE, Messina LM. Relief of mesenteric ischamia in type III aortic dissection with percutaneous fenestration of the aortic septum. Radiology $1990 ; 174: 450-2$

80. Cowling MG, Redwood D, Buckenham TM. Case report: critical lower limb ischaemia due to aortic dissection relieved by percutaneous transfemoral fenestration. Clin Radiol 1995; 50: 654-7.

81. Faykus MH Jr, Hiette P, Koopot R. Percutaneous fenestration of a type I aortic dissection for relief of lower extremity ischaemia. Cardiovasc Intervent Radiol 1992; $15: 183-5$.

82. Gorge G, Erbel R. [Intravascular ultrasound for monitoring percutaneous fenestration of a menbrane from anaortic dissection]. Dtsch Med Wochenschr 1996; 121: 1598-602.

83. Trerotola So. Use of a stone basket as a target during fenestration of aortic dissection. J Vasc Interv Radiol 1996; 7:687-90.

84. Kato N, Sakuma H, Takeda K, Hirano T, Nakagawa T. Relief of acute lower limb ischaemia with percutaneous fenestration of intimal flap in a patients with type III aortic dissection - a case report. Angiology 1993; 44: 755-9.

85. Saito S, Arai H, Kim K, Aoki N, Tsurugida M. Percutaneous fenestration of dissecting intima with a transseptal needle. Cathet Cardiovasc Diagn 1992; 26 : $130-5$.
86. Walker PJ, Dake MD, Mitchell RS, Miller DC. The use of endovascular techniques for the treatment of complications of aortic dissection. J Vasc Surg 1993; 18: 1042-51.

87. Williams DM, Lee DY, Hamilton BH et al. The dissected aorta: percutaneous treatment of ischaemic complications - principles and results. J Vasc Interv Radiol 1997; 8: 605-65.

88. Lee DY, Williams DM, Abrams GD. The dissected aorta. Part II. Differentiation of the true from the false lumen with intravascular US. Radiology 1997; 203:32-6.

89. Moon MR, Michell RS, Dake MD, Zarins CK, Fann JI, Miller DC. Simultaneous abdominal aortic replacement and thoracic stent-graft placement for multilevel aortic disease. J Vasc Surg 1997; 25: 332-40.

90. Elefteriades JA, Hammond GL, Gusberg RJ, Kopf GS, Baldwin JC. Fenestration revisited: a safe and effective procedure for descending aortic dissection. Arch Surg 1990; 125: 786-90.

91. Shennan T. Dissecting aneurysm. Medical Research Council Special. Report Series, $N^{\circ} 193$. London: Her Majesty's Stationery Office, 1984.

92. Nienaber CA, Fattori R, Lund G et al. Nonsurgical reconstruction of thoracic aortic dissection by stent-graft placement. NEngl J Med 199; 340: 1539-45.

93. Dake MD, Miller DC, Mitchell RS, Semba CP, Moore KA, Sakai T. The "first genetarion" of endovascular stent-grafs for patients with aneurysms of the descending thoracic aorta. J Thorac Cardiovasc Surg 1998; 116: 689-703. Discussion 703-4

94. Grabenwöger M, Hutschala D, Ehrlich MP, Cartes-Zumelzu F et al. Thoracic aortic aneurysms treatment with endovascular self-expandable stents grafs. Ann Thorac Surg 2000; 69: 441-5.

95. Fann J \& Miller C. Endovascular treatment of descending thoracic aortic aneurysms and dissections. Surg Clin Nort Am 1999; 79: 551-74.

96. Plama JH, Souza JAM, Alves CMR, Carvalho AC. Self-expandable aortic stentgrafts for treatment of descending aortic dissections. Ann Thorac Surg 2002; 73 : 1138-42.

97. Buffolo E, Fonseca JHP, Souza JAM, Alves CMR. Revolutionary treatment of anuerysms and dissections of descending aorta: the endovascular approach. Ann Thorac Surg 2002; 74: S1815-7.

98. Guthaner DF, Miller DC, Silverman JF et al. Fate of the false lumen following surgical repair of aortic dissections. An angiographic study. Radiology 1978; 122: 1-8.

99. Erbel R, Oelert H, Meyer J et al. Influence of medical and surgical therapy on aortic dissection evaluated by transophageal echocardiography. Circulation 1993; 87: 1604-15.

100. Di Cesare E, Di Renzi P, Pavone P, Marsili L, Castallo F, Parassariello R. Postosurgical follow-up of aortic dissections by MRI. Eur J Radiol 1991; 13: 27-30.

101. Hara Km Yamaguchi T, Wanibuchi Y, Kurokawa K. The role of medical treatment of distal type aortic dissection. Int J Cardiol 1991;32:231-40.

102. Masani ND, Banning AP, Jones RA, Tuttley MS, Fraser AG. Follow-up of chronic thoracic aortic dissection: comparison of transesophageal echocardiography and magnetic resonance imaging. Am Heart J 1996; 131: 1156-63.

103. Ergin MA, Phillips RA, Galla JD et al. Significance of distal false lumen after type A dissection repair. Ann Thorac Surg 1994;57:820-5.

104. Neufang KF, Theisen P, DeiderS, SechtemU. Thoracic aorta dissection-the place of MRT and CT in the follow-up after prosthetic aortic replacement. Fortschr Rontgenstr 1989; 151:659-65.

105. Rizzo JA, Darr U, Fischer M et al. Mutimodality serial follow-up of thoracic aortic aneurysms. Int J Angiol 1997; 6: 153-6.

106. DeBakey ME, McCollum CH, Crawford ES et al. Dissection and dissecting aneurysms of the aorta: Twenty-year follow-up of five hundred twenty-seven patients treated surgically. Surgery 1982;92:1118-34.

107. Miller DC, Mitchell RS, Oyer PE et al. Independent determinants of operative mortality for patients with aortic dissections. Circulation 1984 (suppl 1): 70: 153-64.

108. Glower DD, Speier RH, White WD, Smith LR, Rankin JS, Wolfe WG. Management and longterm outcome of aortic dissection. Ann Surg 1991;214;31-41.

109. Crawford ES, Coselli JS. Marfan's syndrome: Combined composite valve graft replacement of the aortic root and transaortic mitral valve replacement. Ann Thorac Surg 1988; 45: 296-302.

110. McDonald GR, Schaff HV, Pyeritz RE, McKusick VA, Gott VL. Surgical management of patients with the Marfan syndrome and dilatation of the ascending aorta. J Thorac Cardiovasc Surg 1981;81: 180-6.

111. American Academy of Pediatrics Committee on Genetics. Heath supervision for children with Marfan's syndrome. Pediatrics 1996; 98:978-82.

112. Taylor JFN. Clinical pediatric cardiology. Curr Opin Cardiol 1993; 8: 108-13.

113. Braverman AC. Exercise and the Marfan syndrome. Med Sci Sports Exerc 1998; 30:387-95.

114. Milewicz DM, Michael K, Fisher N, Coselli J, Markello T, Biddinger A. Fikbrillin-1 (FBN1) mutations in patients whit thoracic aortic aneurysms. Circulation 1996; 94: 2708-11.

115. Alfonso F, Goicolea J, Aragoncillo P, Hernandez R, Macaya C. Diagnosis of 
aortic intramural hematoma by intravascular ultrasound imaging. Am J Cardio $1995 ; 76: 735-8$

116. Ide K, Uchida H, Otsuji Het al. Acute aortic dissection with intramural hematoma: possibility of transition to classic dissection or aneurysm. J Thorac Imaging 1996; 11:46-52

117. Nienaber CA, von Kodolitsch Y, Petersen B et al. Intramural hemorrhage of the thoracic aorta. Diagnostic and therapeutic implications. Circulation 1995;92: 1465-72.

118. Murray JG, Manisali M, Flamm SD et al. Intramural hematoma of the thoracic aorta: MR image findings and their prognostic implications. Radiol 1997; 204 : 349-55.

119. Bolognesi R, Manca C, Tsialtas Det al. Aortic intramural hematoma: an increasingly recognized aortic disease. Cardiology 1998; 89: 178-83.

120. Kaji S, Nishigami K, Akasaka Tet al. Prediction of progression or regression of type A aortic intramural haematoma by computed tomography. Circulation 1999; 100: II $281-6$

121. Pepi M, Campodonico J, Galli C et al. Rapid diagnosis and management of thoracic aortic dissection and intramural haematoma: a prospective study of advantages of multiplane vs. biplane transesophageal echocardiography. Eur JEchocardiography $2000 ; 1: 72-9$.

122. Vilascosta I, San Roman JA, Ferreiros J, Aragoncillo P, Mendez R et al. Natural history and serial morphology of aortic intramural haematoma: a novel variant of aortic dissection. Am Heart J 1997; 134: 495-507.

123. Cooke JP, Kazmier FJ, Orszulak TA. The penetrating aortic ulcer: pathologic manifestations, diagnosis and management. Mayo Clin Proc 1988; 63: 718-25.

124. Kazerooni EA, Bree RL, Williams DM. Penetrating atherosclerotic ulcers of the descending thoracic aorta: evaluation with $\mathrm{CT}$ and distinction from aortic dissection. Radiology 1992; 183: 759-65.

125. Coady MA, Rizzo JA, Hammond GL, Pierce JG, Kopf GS, Elefteriades JA Penetrating ulcer of the thoracic aorta: what is it? How do we recognize it? How so we mange it? J Vasc Surg 1998; 27: 1006-16.

126. Coady MA, Rizzo JA, Elefteriades JA. Pathologic variants of thoracic aortic dissections. Penetrating atherosclerotic ulcers and intramural haematomas. Cardio Clin 1999; 17: 637-57.

127. Hussain S, Glover JL, Bree R, Bendick PJ. Penetrating atherosclerotic ulcers of the thoracic aorta. J Vasc Surg 1989; 9: 710-7.

128. Gorenink M, Rozendaal L, Naeff MS et al. Marfan syndorme in children and adolescent: predictive and prognostic value of aortic root growth for screening for aortic complications. Heart 1998; 80: 163-9.

129. Gott VL, Geene PS, Alejo DEet al. replacement of the aortic root in patients with Marfan's syndrome. NEngl J Med 1999; 340: 1307-13

130. Finkbohner R, Johnston D, Crawford ES, Coselli J, Milewicz DM. Marfan syndrome. Long-term survival and complications after aortic aneurysm repair. Circulation 1995; 91: 728-33.

131. Silverman DI, Burton KJ, Gray J et al. Life expectancy in the Marfan syndrome. Am JCardiol 1995; 75: 157-60

132. Legget ME, Unger TA, O'Sullivan CK et al. Aortic root complications in Marfan's syndrome: identification of a lower risk group. Heart 1996; 75: 389-95.

133. Davies RR, Goldstein LJ, Coady MA, Tittle SL, Rizzo JA et al. Yearly rupture or dissection rates for thoracic aortic aneurysms: simple prediction based on size. Ann Thorac Surg 2002; 73: 17-28.

134. Carrel T, Von Segsser L, Jenni Ret al. Dealing with dilated ascending aorta during aortic valve replacement: advantages of conservative surgical approach. Eur J Cardiothorac Surg 1991; 15: 137-43.

135. Yun KL, Miller DC, Fann JI et al. Composite valve graft versus separate aortic valve and ascending aortic replacement: is there still a role for the separate procedure. Circulation 1997; 96 (suppl 1): 368-75

136. David TE. Current practice in Marfan's aortic root surgery: reconstruction with aortic valve preservation or replacement? What to do with the mitral valve? J Card Surg 1997; 12 (suppl 2): 147-50.

137. Coady MA, Rizzo JÁ, Hamond GL et al. What is the appropriate size criterion for resection of thoracic aortic aneurysms? J Thorac Cardiovasc Surg 1997; 113 : 476-91

138. Pyritz RE. Marfan's syndrome: Current and future clinical and genetic management of cardiovascular manifestations. Semin Thorac Cardiovasc Surg 1993; 5 : 11-6.

139. Hwa J, Richards JG, Huang $\mathrm{H}$ et al. The natural history of aortic dilatation in Marfan's syndrome. Med J Aust 1993; 158: 558-62.

140. Juvonen T, Ergin MA, Galla JD et al. Prospective study of the natural history of thoracic aortic aneurysms.Ann Thorac Surg 1997; 63:1533-45.

141. Griepp RB, Ergin A, Galla JD, Lansman SL, McCullough JN et al. Natural history of descending thoracic and thoracoabdominal aneurysms. Ann Thorac Surg 1999; 67: 1927-30.

142. Coady MA, Rizzo JA, Hammong GLet al. What is the appropriate size criterion for resection of thoracic aortic aneurysms? J Thorac Cardiovasc Surg 1997; 113 476-91.
143. Coady MA, Davies RR, Roberts M et al. Familial patterns of thoracic aortic aneurysms. Arch Surg 1999; 134:361-7.

144. Goldstein LJ, Davies RR, Davilla JJ et al. Stroke in thoracic aortic surgery: incidence, impact, etiology, and prevention. J Thorac Cardiovasc Surg 2001; 122: $935-45$

145. Cambria RO, Davison JK, Carter C et al. Epidural cooling of spinal cord protection during thoracoabdominal aneurysms repair: a five year experience. J Vasc Surg 2000; 31: 1093-102.

146. Grabitz K, Sandmann W, Stuhmeier Ket al. The risk of ischemic spinal cord injury in patients undergoing graft replacement for thoracoabdominal aortic aneurysms. J Vasc Surg 1996; 23: 230-40.

147. Coselli JS. Recent advances in surgical treatment of thoracoabdominal aortic aneurysms. In: Chiesa R, Melissano G. Gli Aneurismi dell’Aorta Addominale. Milano: Europa Scienze Umane Editrice; 1996. p. 269-84.

148. Acher CW, Wynn MM, Hoch JR, Popic P, Archibald J, Turnipseed WD. Combined use of cerebral spinal fluid drainage and naloxone reduces the risk of paraplegia in thoracoabdominal aneurysm repair. J Vasc Surg 1994; 19: 236-48.

49. Svensson LG, Crawford ES, Hess KR, Coselli JS, Safi HJ. Experience with 1509 patients undergoing thoracoabdominal aortic operations. J Vasc Surg 1993; 17 $357-70$.

150. HollierLM, Money S, Naslund Tetal.Risk of spinal cord dysfunction in patients undergoing aortic replacements. Am J Surg 1992; 164: 201-4.

151. Safi HJ, Campbell MP, Miller IIICC et al. Cerebral spinal fluid drainage and dista aortic perfusion decrease the incidence of neurological deficit: the results of 343 descending and thoracoabdominal aortic aneurysm repairs. Eur J Vasc Endovasc Surg 1997; 14: 118-24.

152. Coselli JS, LeMaire AS. Left heart bypass reduces paraplegia rates after thoracoabdominal aortic aneurysm repair. Ann Thorac Surg 1999; 67: 1931-4.

153. Schepens M, Defauw J, Hamerlijnck R, VermeulenF. Use of left heart bypass in the surgical repair of thoracoabodminal aortic aneurysms. Ann Vasc Surg 1995; 9 : $327-38$.

154. Svensson LG, Stewart RW, Cosgrove DM $3{ }^{\text {rd }}$ et al. Intrathecal papaverine for the prevention of paraplegia after operation in the thoracic or thocoabdominal aorta. J Thorac Cardiovas Surg 1998; 96: 823-9.

155. Svensson LG, Crawford ES, Hess KR, Coselli JS, Safi HJ. Thoracoabdomina aortic aneurysms associated whit celiac, superior mesenteric, and renal artery occlusive disease: methods and analysis of results in 271 patients. J Vasc Surg 1992; 16:378-90

156. Cooley DA, Golino A, Frazier OH. Single-clamp technique for aneurysms of the descending thoracic aorta: report of 132 consecutive cases. Eur J Cardiothorac Surg 2000; $18: 162-7$.

157. Crawford ES, Crawford JL, Safi HJ et al. Thoracoabdominal aortic aneurysms: Preoperative and intraoperative factors determining immediate and long-term results of operation in 605 patients. J Vasc Surg 1986; 3: 389-404

158. Crawford ES, Coselli JS, Safi HJ. Partial cardiopulmonary bypass, hypothermic circulatory arrest, and posterolateral exposure for thoracic aortic aneurysm operation. J Thorac Cardiovasc Surg 1987;94: 824-7.

159. Verdant A, Cossette R, Page A et al. Aneurysms of the descending thoracic aorta Three hundred sixty-six consecutive cases resected without paraplegia. J Vasc Surg 1995;21:85-91.

160. Miyamoto K, Keno A, Wada T et al. A new and simple method of preventing spinal cord damage following temporary occlusion of the thoracic aorta by draining the cerebrospinal fluid. J Cardiovasc Surg 1960;16:188-99.

161. Blaisdell FW, Cooley DA. The mechanism of paraplegia after temporary thoracic aortic occlusion and its relationship to spinal fluid pressure. Surgery 1962;51: $351-5$.

162. Wadouh F, Lindemann EM, Arndt CFet al. The arteria radicularis magna anterior as a decisive factor influencing spinal cord damage during aortic occlusion. JThorac Cardiovas Surg 1984; 88: 1-10.

163. Svensson LG, Von RitterCM, Broeveld HT et al. Cross-clamping of the thoracic aorta: Influence of aortic shunts, laminectomy, papaverine, calcium channel blockers, allopurinol, ad superoxide dismutase on spinal cord blood flow and paraplegia in baboons. Ann Surg 1986; 204: 38-47

164. Kazama S, Masaki Y, Maruyama S, Ischihara A. Effects of altering cerebrospina fluid pressure on spinal cord blood flow. Ann Thorac Surg 1994;58: 112-5.

165. Simpson JI, Eide TR, Schiff GA et al. Intrathecal magnesium sulfate protects the spinal cord from ischemic injury during thoracic aortic cross-clamping. Anesthesiology 1994; 81: 1493-9.

166. Follis F, Miller K, Scremin OU et al. NMDA receptor blockade and spinal cord ischemia due to aortic crossclamping in the rat model. Can J Neurol Sci 1994; 21 : 227-32

167. Maughan RE, Mohan C, Nathan IMet al. Intrathecal perfusion of an oxygenated perflurocarbon emulsion prevents paraplegia after extended normothermic aortic cross-clamping. Ann Thorac Surg 1992; 54: 818-24

168. Murray MJ, Bower TC, Oliver WCJ et al. Effects of cerebrospinal fluid drainage in patients undergoing thoracic and thoracoabdominal aortic surgery. J Cardiothorac Vasc Anesth 1993; 7:266-72. 
169. Acher CW, WynnMM, Hoch JR et al. Combined use of spinal fluid drainage and naloxone reduces risk of neurologic deficit in the repair of thoracoabdominal aneurysms. J Vasc Surg 1994; 19: 236-48.

170. Safi HJ. Neurologic deficit in patients at high risk with thoracoabdominal aortic aneurysms: The role of cerebral spinal fluid drainage and distal aortic perfusion. JVas Surg 1994; 20: 434-43.

171. Svensson LG, Patel V, Robinson MFet al. Influence of preservation or perfusion intraoperatively identified spinal cord blood supply on spinal motor evoked potentials and paraplegia after aortic surgery. J Vasc Surg 1991; 13: 355-65.

172. Svensson LG, Stewart RW, Cosgrove DM3 et al. Intrathecal papaverine for the prevention of paraplegia after operation on the thoracic or thoracoabdominal aorta. J Thorac Cardiovasc Surg 1988; 96: 823-9.

173. Svensson LG, Grum DF, Bednarski M et al. Appraisal of cerebrospinal fluid alterations during aortic surgery with intrathecal papaverine administration and cerebrospinal fluid drainage. JVasc Surg 1990; 11:423-9.

174. Svensson LG, Crawford ES, Hess KR et al. Experience with 1509 patients undergoing thoracoabdominal aortic operations. J Vasc Surg 1993; 17: 357-70

175. Mutch WA, Graham MR, Halliday WC et al. Use of neuroanesthesia adjuncts hyperventilation and mannitol administration improves neurological outcome after thoracic aortic cross-clamping in dogs. Stroke 1993; 24: 1204-10.

176. Grabitz K, Freye E, Prior R et al. Does prostaglandin E1 and superoxide dismutase prevent ischaemic spinal cord injury after thoracic aortic crossclamping? Eur J Vasc Surg 1990; 4: 19-24

177. Svensson LG, Loop FD. Prevention of spinal cord ischemia in aortic surgery, in Bergan JJ, Yao JST. Arterial Surgery: New Diagnostic and Operative Techniques. NY, Grune \& Stratton, 1988,pp. 273-85

178. Johnson SH, Kraimer JM, Graeber GM. Effects of flunarizine on neurological recovery and spinal cord blood flow in experimental spinal cord ischemia in rabbits. Stroke 1993; 24: 1547-53.

179. Svensson LG. New and future approaches for spinal cord protection. Semin Thorac Cardiovas Surg 1997; 9: 206-21.

180. Dommisse GF. The blood supply of the spinal cord. J Bone Joint Surg (Br) 1974; 56:225-35.

181. Svensson LG, Klepp P, Hinder RA. Spinal cord anatomy of the baboon: comparison with man and implications on spinal cord blood flow during thoracic aortic cross-clampintg. S Afr Surg 1986;24:32-4

182. Dommisse GF. The Arteries and Veins of the Human Spinal Cord from Birth Edinburgh, Churchill Livingstone, 1975 .

183. Svensson LG, Hess KR, Coselli JS, Safi HR. Influence of segmental arteries, extent, and atrio-femoral bypass on postoperative paraplegia after thoracoabdominal aortic aneurysm repairs. J Vasc Surg 1994; 20: 255-62

184. Williams GM. Treatment of chronic expanding dissecting aneurysms of the descending thoracic and upper abdominal aorta by extended aortotomy, removal of the dissected intima, and closure. J Vasc Surg 1993; 18:441-9.

185. Svensson LG. Intraoperative identification of spinal cord blood supply during descending and thoracoabdominal aortic repairs. J Thorac Cardiovasc Surg 1997; 112: 1455-61

186. Svensson LG, Richards E, Coull A et al. Relationship of spinal cord blood flow to vascular anatomy during thoracic aortic cross-clamping and shunting. JThorac Cardiovasc Surg 1986;91:71-8.

187. Crawford ES, Mizrahi EM, Hess KR et al. The impact of distal aortic perfusion and somatosensory evoked potential monitoring on prevention of paraplegia after aortic aneurysm operation. J Thorac Cardiovasc Surg 1988; 95: 357-67.

188. Svensson LG, Crawford ES, Patel V et al. Spinal cord oxygenation, intraoperative blood supply localization, cooling and function with aortic clamping. Ann Thorac Surg 1992; 54: 74-9.

189. Coselli JS, LeMaire SA, Buket S, Berzin E. Subsequent proximal aortic operations in 124 patients with previous infrarenal abdominal aortic aneurysm surgery. J Vasc Surg 1995; 22: 59-67.

190. Osenbach RK, Hitchon PW, Mouw L, Yamada T. Effect of spinal cord ischemia on evoked potential recovery and post-ischemic regional spinal cord blood flow. J Spinal Disord 1993; 6: 146-54

191. Reuter DG, Tacker WAJ, Badylak SF, Voorhees WD. Correlation of motor-evoked potential response to ischemic spinal cord damage. J Thorac Cardiovasc Surg 1992; 104:262-72

192. Shokoku S, Uchida H, Teramoto S. An experimental study on spinal cord ischemia during cross-clamping of the thoracic aorta: monitoring of spinal cord ischemia with motor evoked potential by transcranial stimulation of the cerebral cortex in dogs. Surg Today 1993; 23: 1068-73.

193. Matsui Y, Goh K, Shiiya N et al. Clinical application of evoked spinal or potentials elicited by direct stimulation of the cord during temporary occlusion of the thoracic aorta. J Thorac Cardiovasc Surg 1994; 107: 1519-27.

194. Dake MD, Miller DC, Semba CP et al. Transluminal placement of endovascular stent-grafts for the treatment of descending thoracic aortic aneurysms. N Eng JMed 1994; 331: 1729-34.

195. Dake MD, Miller DG, Mitchell RS, Semba CP, Moore KA, Sakai T. The "first generation" of endovascular stent-grafts for patients with aneurysms of the descending thoracic aorta. J Thorac Cardiovasc Surg 1998; 116: 689-704.
196. Leopold GR, Goldberger LE, Bernstein EF. Ultrasonic detection and evaluation of abdominal aortic aneurysms. Surgery 1972;72:939-45.

197. Nehler MR, Taylor LM, Moneta GL, Porter JM. Indications for operation for infrarenal abdominal aortic aneurysms: Current Guidelines. Seminars in Vascular Surgery $1995 ; 8: 108-14$

198. Thurmond A. Abdominal aortic aneurysm: Incidence in a population at risk. J Cardiovasc Surg 1986; 27: 457-9.

199. MacSweeney STR, O'Meara M, Alexander C et al. High prevalence of unsuspected abdominal aortic aneurysm in patients with confirmed symptomatic peripheral or cerebral arterial disease. BrJ Surg 1993; 80:582-4.

200. Taylor LM Jr, Porter JM. Abdominal aortic aneurysms. Ann Vasc Surg 1987; 1 : 502-4.

201. Glimaker H, Holmberg L, Elvin A et al. Natural history of patients with abdominal aortic aneurysm. Eur J Vasc Surg 1991; 5: 125-30.

202. Donaldson MC, Rosenberg JM, Bucknam CA. Factors affecting survival after ruptured abdominal aortic aneurysm. J Vasc Surg 195; 2: 564-70.

203. Nevitt MP, Ballard DJ, Hallet JW Jr. Prognosis of abdominal aortic aneurysms: A population-based study. NEngl J Med 1989; 321: 1009-14.

204. Reigel MM, Hollier LH, Kazmier FJ et al. Late Survival in abdominal aortic aneurysm patients: the role of selective myocardial revascularization on the basis of clinical symptons. J Vasc Surg 1987; 5: 222-7.

205. Hollier LH, Taylor LM Jr., Ochsner J. Recommended indications for operative treatment of abdominal aortic aneurysms. J Vasc Surg 1992; 15: 1046-56.

206. Cronenwett JL, Murphy TF, Zelenock GB et al. Actuarial analysis of variables associated with rupture of small abdominal aortic aneurysms. Surgery $185 ; 98$ : 472-83.

207. Hoffman M, Avellone JC, Plecha FR et al. Operations for ruptured abdominal aortic aneurysms: A community-wide experience. Surgery 1982;91:597-602.

208. Craig SR, Wilson RG, Walker AJ, Murie JA. Abdominal aortic aneurysm: still missing the message. Br J Surg 1993; 80: 450-2.

209. Bernstein EF, Chan EL. Abdominal aortic aneurysm in high-risk patients. Ann Surg 1984;200: 255-63.

210. Donaldson MC, Rosenberg JM, Bucknam CA. Factors affecting survival after ruptured abdominal aortic aneurysm. J Vasc Surg 1985; 2: 564-70

211. Johnston KW, Scobie TK. Multicenter prospective study of nonruptured abdominal aortic aneurysms. Population and operative management. JV Vasc Surg 1988; 7:69-81.

212. Perry MO, Calcagno D. Abdominal aortic aneurysm surgery: The basic evaluation of cardiac risk. Ann Surg 1988; 208: 738-42.

213. Green RM, Ricotta JJ, Ouriel K, DeWeese JA. Results of supraceliac aortic clamping in the difficult elective resection of intrarenal abdominal aortic aneurysm. J Vasc Surg 1989; 9: 124-34

214. Leather RP, Shah DM, Kaufman JLet al. Comparative analysis of retroperitoneal aortic replacement for aneurysm. Surg Gynecol Obstet 1989; 168: 387-93.

215. Sicard GA, Allen BT, Munn JS, Anderson CB. Retroperitoneal versus transperitoneal approach for repair of abdominal aortic aneurysms. Surg Clin Nort Am 1989; 69: 795-806

216. Sullivan CA, Rohrer MJ, Cutler BS. Clinical management of the symptomatic but unruptured abdominal aortic aneurysm. J Vasc Surg 1990; 11: 799-803.

217. AbuRahma AF, Robinson PA, Boland JP et al. Elective resection of 332 abdominal aortic aneurysms in a southern West Virginia community during a recent fiveyear period. Surgery; 1991: 109:244-51.

218. Hannan EL, O'Donnell JF, Kilburn H et al. Investigation of the relationship between volume and mortality for surgical procedures performed in New York State hospitals. JAMA 1989;262: 503-10.

219. The United Kingdom Small Aneurysm Trial Participants. Long-term outcomes of immediate repair compared with surveillance of small abdominal aortic aneurysms. NEng J Med 2002; 346: 1445-52.

220. Lederle FA, Wilson SE, Johnson GR, Reinke DB, Littooy FN et al. Immediate repair compared whit surveillance of small abdominal aortic aneurysms. NEng J Med 2002;346: 1437-44.

221. Lederle FA, Jonson GR, Wilson SE, Ballard DJ, Blebea J et al. Rupture rate of large abdominal aortic aneurysms in patients refusing pr unfit for elective repair. JAMA 2002; 287: 2968-72.

222. Parodi JC, Palmaz JC, Barone HD. Transfemoral intraluminal graft implantation for abdominal aortic aneurysms. Ann Vasc Surg 1991; 5: 491-9.

223. Hallett JW Jr. Management of abdominal aortic aneurysms. Mayo Clin Proc 2000; 75:395-9.

224. Hallet JW Jr, Marshall DM, Petterson TM et al. Graft-related complications after abdominal aortic aneurysm repair: reassurance from a 36-year population-based experience. J Vasc Surg 1997; 25: 277-84.

225. May J, Woodburn K, White G. Endovascular treatment of infrarenal abdominal aortic aneurysms. Ann Vasc Surg 1998; 12:391-5

226. Brewster DC, Cronenwett JL, Hallet JW et al. Guidellines for the treatment of abdominal aortic aneurism. Report of a subcommitee of the Joint Council of the American Association for Vascular Surgery and Society for Vascular Surgery. J Vasc Surg 2003; 37: 1106-17. 